\title{
On the locus of the effect of alerting on response conflict: An event-related EEG study with a speed-accuracy tradeoff manipulation
}

\author{
Dariusz Asanowicz*, Kinga Wołoszyn, Bartłomiej Panek, Eligiusz Wronka \\ Institute of Psychology, Jagiellonian University, Krakow, Poland
}

\section{A R T I C L E I N F O}

\section{Keywords:}

Attention

Response conflict

Executive control

Alerting

Flanker task

ERPs

Midfrontal theta

Speed-accuracy tradeoff

\begin{abstract}
A B S T R A C T
The present study investigated the locus of the effect of alerting on response conflict, and examined whether this effect may be interpreted as an alerting-triggered imbalance of speed-accuracy tradeoff (SAT). Participants performed the flanker task with an alerting tone in half of the trials and SAT manipulation. Behavioral results showed the usual increase of the conflict effect (incongruent-congruent flankers) in the alerting trials. This interaction was not affected by SAT manipulation in response times, although accuracy emphasis abolished alerting effects in error rates. Event-related potential (ERP) results showed that alerting increased the conflict by facilitating the selection and activation of stimulus-response links, reflected in modulations of the P3b component, and by enhancing the activation of incorrect response evoked by incongruent flankers, reflected in an increased initial incorrect activation in the lateralized readiness potential (LRP). Time-frequency analysis showed that the alerting-triggered increase of conflict entailed stronger response of executive mechanisms, reflected in a larger conflict-related midfrontal theta-band power. These EEG effects were not affected by SAT manipulation. In conclusion, alerting affects both the emergence of conflict and conflict control, and this alerting-conflict interaction could not be explained in terms of SAT.
\end{abstract}

\section{Introduction}

The present study had a twofold aim: to investigate the locus of the effect of attentional alerting on response conflict in the flanker task, and to examine whether this effect may be interpreted and possibly explained in terms of speed-accuracy tradeoff (SAT).

\subsection{Conflict resolution and attentional alerting}

Conflict resolution is a paradigm case for studying mechanisms underlying our ability of effortful control and self-regulation (Botvinick, Braver, Barch, Carter, \& Cohen, 2001; Posner \& Rothbart, 2007), and has been investigated using a range of experimental conflict tasks of which most common are variations of the Stroop task (Stroop, 1935), the Simon task (Simon, 1969), and the flanker task (Eriksen \& Eriksen, 1974). In each of these tasks, a conflict or interference is induced by manipulating stimulus-response compatibility and measured by comparing participants' performance in compatible and incompatible trials (see, e.g., Egner, 2008 for a detailed description of these tasks). In the flanker task, a trial consists of a target stimulus (e.g., a left- or rightpointing arrow), which requires specific manual response (e.g., pressing a button with the left or right hand, respectively to the left and right arrows), and flanker stimuli, which are either congruent with the target (e.g., target and flankers are all left arrows) or incongruent (e.g., the target is a left arrow and flankers are right arrows). Thus, two fixed stimulus-response (S-R) links, or "event-files" (Hommel, 2004), are established by instruction and practice (e.g., left arrow = left-hand response, right arrow = right-hand response). Consequently, in the incongruent condition, the target and flankers simultaneously activate both of these established S-R links, triggering a conflict between the two response programs (Egner, 2008; Miller, 1991). Resolution of this conflict entails costs reflected in slower and less accurate responses. The difference between the two flanker conditions, i.e., the conflict effect (incongruent-congruent), is interpreted as an index of the efficiency of executive control in conflict detection and resolution (the higher the efficiency, the smaller the conflict effect) (Egner, 2008; Posner \& Rothbart, 2007).

This conflict effect somewhat paradoxically increases when the target is preceded by an accessory stimulus, e.g., a short tone or visual cue occurring about few hundred ms before target onset (Asanowicz \& Marzecová, 2017; Callejas, Lupiáñez, \& Tudela, 2004; Fossella et al., 2002; Ishigami \& Klein, 2010). The accessory stimulus triggers exogenous phasic alerting, i.e., a short-lived enhancement of readiness of perceptual systems to process incoming external stimuli (Hackley \&

\footnotetext{
* Corresponding author at: Institute of Psychology, Jagiellonian University, Ingardena 6, 30-060 Krakow, Poland.

E-mail address: asanowicz@uj.edu.pl (D. Asanowicz).
} 
Valle-Inclán, 2003; Posner, 2008), which increases the speed of behavioral responses. Behavioral, electrophysiological, and imaging studies suggest that this response facilitation is produced by increasing the speed of processing and lowering the threshold at several stages of the S-R pathway, from visual processing and perceptual discrimination (Böckler, Alpay, \& Stürmer, 2011; Fischer, Plessow, \& Ruge, 2013; Kusnir, Chica, Mitsumasu, \& Bartolomeo, 2011; Petersen, Petersen, Bundesen, Vangkilde, \& Habekost, 2017; Wiegand et al., 2017) to decision making and early phases of response selection (Böckler et al., 2011; Hackley \& Valle-Inclán, 1998, 1999; Hackley et al., 2009; Yanaka, Saito, Uchiyama, \& Sadato, 2010; Yoshida et al., 2013). Of importance, this alerting-induced improvement is usually smaller in the incongruent than in the congruent flanker condition, resulting in the aforementioned larger conflict effect in trials with alerting stimuli.

Several accounts of the negative impact of alerting on conflict resolution have been put forward. One bone of contention between them is the locus of the interaction: On what stage or level of information processing this interaction of alerting and response conflict occurs? Weinbach and Henik (2012) have proposed that alerting enhances global processing of visual stimuli, which leads to allocation of more visual attention to the irrelevant spatial information, such as the incongruent flankers (the global processing hypothesis). Nieuwenhuis and de Kleijn (2013), on the other hand, have argued that alerting reduces the time of stimulus encoding, thereby accelerating the onset of perceptual decisions, which in turn reduces the time available for effective conflict resolution and proper response selection. Thus, alerting interacts with conflict processing at the level of perceptual decision making (the early onset hypothesis). Another account, proposed by Fischer, Plessow, and Kiesel, 2010; Fischer, Plessow, \& Kiesel, 2012, locates the interaction at later, post-perceptual stages of visuomotor integration and response selection (the response selection hypothesis). They have suggested that accessory stimuli facilitate the activation of established S-R links, thereby accelerating the transfer of stimulus codes into corresponding response codes. In consequence, alerting increases the probability of activation of an incorrect response via a direct, reflexlike S-R route (cf. the dual-route models, e.g., De Jong, Liang, \& Lauber, 1994; Eimer, Hommel, \& Prinz, 1995). Finally, Posner (1994, 2008) has suggested that alerting may directly affect the mechanism of conflict detection and resolution. In order to facilitate rapid responding to external events (e.g., a warning signal), phasic alerting suppresses ongoing executive processes, thereby decreasing the efficiency of conflict processing (the suppression hypothesis; see also Callejas, Lupiáñez, Funes, \& Tudela, 2005; Petersen \& Posner, 2012). In summary, the effect of alerting on response conflict may occur prior to the emergence of conflict, either at the level of the global visuospatial processing, or the perceptual decisions, or the activation of S-R links, or else already at the level of conflict detection and resolution, due to the suppression of executive control.

In the present study, to further investigate the locus of the effect of alerting on response conflict, we recorded electroencephalogram (EEG) while participants performed the arrow flanker task with a phasic alerting manipulation (trial-wise; an auditory alerting stimulus was presented $100 \mathrm{~ms}$ before the target in half of the trials). Of interest were three EEG event-related potentials (ERPs), the N1 component of early visual potential, the P3b component of target-evoked potential, and the lateralized readiness potential (LRP), along with conflict-related midfrontal EEG oscillatory activity in the theta frequency range.

The visual N1 is a negative peak occurring about $180-200 \mathrm{~ms}$ after stimulus onset at sites located above the occipito-temporal cortex (usually PO7 and PO8). This component reflects perceptual processes of stimulus encoding and discrimination (Hopf, Vogel, Woodman, Heinze, \& Luck, 2002; Vogel \& Luck, 2000). The P3b component is a positive deflection with a maximum at parietal midline (usually $\mathrm{Pz}$ ) at about $300-700 \mathrm{~ms}$ after target onset. Results obtained by Verleger and colleagues suggest that $\mathrm{P} 3 \mathrm{~b}$ may reflect processes of S-R translation (Verleger, Jaśkowski, \& Wascher, 2005; for similar conclusions see
Pritchard, Houlihan, \& Robinson, 1999; Valle-Inclán, 1996) and activation of established S-R links (Verleger, Metzner, Ouyang, Śmigasiewicz, \& Zhou, 2014; Verleger, Asanowicz, Werner, \& Śmigasiewicz, 2015; Verleger, Hamann, Asanowicz, \& Śmigasiewicz, 2015). Accordingly, P3b may be used to measure the effects of flanker conflict on activation of S-R links. Indeed, in the incongruent trials, P3b is often smaller and/or delayed, compared to the congruent trials (Kałamała, Szewczyk, Senderecka, \& Wodniecka, 2017; Kałamała, Drożdżowicz, Szewczyk, Marzecová, \& Wodniecka, 2018; Neuhaus et al., 2010; Osman et al., 2000; Smid, Mulder, \& Mulder, 1990; similar effects are observed in other response-conflict procedures like the Simon task, see Verleger, 1997, for a review), which is in line with the assumption that response conflict delays the activation of the correct S-R link. The LRP is the contralateral-ipsilateral (to the responding hand) difference in activity recorded at the sites located over the right and left motor cortex (usually C3 and C4, or C ${ }^{\prime}$ and $\mathrm{C}^{\prime}$ ), and may be used as an index of selective response activation (Eimer, 1998; Smulders \& Miller, 2012). In the congruent trials of the flanker task, LRP emerges about $200-400 \mathrm{~ms}$ after target onset, reflecting activation of the correct response. In the incongruent trials, LRP usually shows premature initial activation of the incorrect response, which peaks at about $300 \mathrm{~ms}$ after the onset of incongruent flankers and is subsequently overruled by delayed activation of the correct response. This reverse from the incorrect to correct response activation presumably is a result of executive control implemented within the premotor brain systems (Gratton, Coles, \& Donchin, 1992; Verleger, Kuniecki, Möller, Fritzmannova, \& Siebner, 2009). The conflict-related midfrontal theta is a specific modulation of ongoing theta-band oscillations ( $4-8 \mathrm{~Hz}$ ), evoked by response conflict, observed at fronto-central sites (usually FCz) about 300-600 ms after stimulus onset. A body of evidence suggests that this effect reflects a neural oscillatory mechanism underlying the processes of conflict detection and resolution (for review see Cavanagh \& Frank, 2014; Cohen, 2014a). The theta modulation originates from the medial prefrontal cortex (mPFC), including the midcingulate cortex (MCC) and the anterior cingulate cortex (ACC) (Nigbur, Ivanova, \& Stürmer, 2011; Cohen, 2011; Pastötter, Hanslmayr, \& Bauml, 2010), and presumably forms a prefrontal-theta network of functional local communication between $\mathrm{mPFC}$ and the areas of dorsal and ventral lateral prefrontal cortex (lPFC) (Cavanagh, Cohen, \& Allen, 2009; Cohen \& Ridderinkhof, 2013), which constitutes the brain's executive control system (cf. Petersen \& Posner, 2012; Ridderinkhof, Ullsperger, Crone, \& Nieuwenhuis, 2004).

If the interaction between phasic alerting and response conflict is due to alerting-triggered enhancement of the scope of visual processing (the global processing hypothesis), the interactive effects should presumably be observed beginning from the visual N1 component. If the interaction is due to alerting-triggered acceleration of stimulus encoding, speeding up the perceptual and, consequently, response decision making (the early onset hypothesis), it should be reflected in shorter latencies of $\mathrm{P} 3 \mathrm{~b}$ (assuming that P3b reflects the S-R translation process, which includes decisions about the target; cf. Twomey, Murphy, Kelly, \& O'Connell, 2015) and in quicker onsets of LRPs (assuming that a shorter stimulus encoding time advances the onset of response selection, cf. Hackley \& Valle-Inclán, 1998). However, LRP amplitudes, i.e., the strength of response activation per se, should not be affected by the alerting-conflict interaction. Further, if the interaction is due to the facilitation of activation of S-R links, which amplifies stimulus-triggered automatic response selection (the response selection hypothesis), the impact of alerting on conflict should be reflected in modulations of P3b and LRP components, possibly in terms of both latencies and amplitudes, and particularly, in the enhancement and/or facilitation of the premature activation of the incorrect response evoked by incongruent flankers. Finally, if alerting entails the suppression of executive control mechanisms (the suppression hypothesis), a weaker conflict-related theta power should be observed in the tone trials, compared to the no-tone trials, along with a delayed onset latency of 
the correct (negative) LRPs in the incongruent condition reflecting a less efficient inhibition of the incorrect response. Alternatively, if alerting affects the conflict at the stages prior to conflict detection and resolution, a larger conflict-related theta power burst should be observed in the tone-trials, reflecting a stronger response of executive mechanisms to the increased conflict.

\subsection{Conflict resolution and speed-accuracy tradeoff}

It has been suggested that phasic alerting accelerates responses by destabilizing tradeoff between speed and accuracy (Hackley \& ValleInclán, 2003; Posner, Klein, Summers, \& Buggie, 1973). This tradeoff is an omnipresent process of adjustment of competing demands in our actions. Importantly, emphasis on speed decreases the quality of information processing (Heitz, 2014; Posner, Nissen, \& Klein, 1976). Therefore, the effect of alerting on conflict might be interpreted in terms of SAT. The alerting-triggered speed gain may have a smaller facilitating impact on RTs in the incongruent trials than in the congruent trials because it may not be possible to speed up responses in the condition with difficult response selection to the same extent as in the condition with easy response selection. At the same time, alerting may increase error rate predominantly in the incongruent trials (in the congruent trials, in which response selection is effortless, accuracy is usually not affected by alerting, cf. Asanowicz \& Marzecová, 2017), because decreasing quality of stimulus processing increases susceptibility for triggering an incorrect response by the incongruent flankers.

On the other hand, the interpretation of the effect of alerting on conflict in terms of SAT assumes a direct relationship between overall response speed and the magnitude of conflict, and this assumption may not be valid. Fast responses are indeed more error-prone in responseconflict tasks (Gratton, Coles, Sirevaag, Eriksen, \& Donchin, 1988; Ridderinkhof, van den Wildenberg, \& Wylie, 2012) and the alertingconflict interaction indeed occurs in the context of decreased average RT. Nevertheless, both Fischer et al. (2010) and Nieuwenhuis and de Kleijn (2013) have shown that the alerting-conflict interaction does not depend on this RT decrease. Moreover, while SAT affects accuracy by definition, it is unclear whether it specifically affects conflict resolution per se, and particularly the speed of conflict resolution (as measured by the standard incongruent-congruent subtraction of RTs). van der Lubbe, Jaśkowski, Wauschkuhn, and Verleger (2001) have found positive evidence showing a larger RT conflict effect in the Simon task when participants performed the task under high time pressure, compared to low time pressure. Similar effect was found in the flanker task by Wylie et al. (2009) - the RT conflict was larger in the speed than in the accuracy condition - but this time only in Parkinson patients, who are known to have impaired executive control thus are more susceptible to experimentally induced response conflict (e.g., Verleger et al., 2010). Finally, in the majority of studies, no SAT effects on the flanker conflict in RTs have been found in healthy adults (Osman et al., 2000; Rinkenauer, Osman, Ulrich, Muller-Gethmann, \& Mattes, 2004; Ullsperger, Bylsma, \& Botvinick, 2005; Wylie et al., 2009).

In conclusion, it is unclear whether the effect of alerting on conflict resolution may be interpreted, let alone explained, as the speed-accuracy tradeoff. While producing similar behavioral effects on overall RTs, alerting and SAT may affect the S-R pathway at different stages and/or via different brain mechanisms. Recent imaging and neurophysiological studies have suggested that speed emphasis increases the baseline activity in decision-associative and pre-motor brain areas, thereby decreasing the threshold for response selection and execution (Bogacz, Wagenmakers, Forstmann, \& Nieuwenhuis, 2010; Heit, 2014). Congruently, ERP studies have shown that speed emphasis increases the amplitude of the P3b (cf. Pfefferbaum, Ford, Johnson, Wenegrat, \& Kopell, 1983) and LRP potentials (Sangals, Sommer, \& Leuthold, 2002), and reduces the LRP onset time relative to onsets of both target stimuli (Osman et al., 2000; Rinkenauer et al., 2004) and motor responses (Rinkenauer et al., 2004; van der Lubbe et al., 2001). Therefore, unlike alerting, which affects the stages preceding response execution (see Section 1.1), SAT may modulate the conflict effect by affecting the response-selection and post-decision response-execution stages.

In the present study, participants were instructed to focus on either speed or accuracy of their responses (block-wise) while performing the flanker task. If alerting affects conflict resolution by triggering SAT, then in the no alerting trials, speed emphasis should produce effects similar to the effects of alerting, in both the behavioral and EEG measures. Moreover, in line with the additive factor logic (Sternberg, 1969), we should observe interactions between alerting and SAT. Alternatively, if alerting and SAT affect conflict resolution at different stages and/or via different mechanisms, this should be reflected in distinctive patterns of the SAT-conflict and alerting-conflict interactions, and the effects of alerting and SAT should be additive, not interactive. In this case, based on the aforementioned ERP studies on SAT, speed emphasis should enhance the effects of conflict on the P3b and LRP components (cf. Section 1.1) independently of the alerting.

\section{Methods}

\subsection{Participants}

Thirty-three undergraduate students took part in the experiment in return for course credits. All participants had normal or corrected-tonormal vision and no history of neurological disorders. Informed written consent was obtained before the experiment. Data from three participants were excluded from analysis due to error rates exceeding 2 SDs of the whole group, and data from other three participants were excluded due to large EEG artifacts. Mean age of the final sample $(N=27 ; 20$ females) was 21.5 years $(\mathrm{SD}=3.0)$.

\subsection{Experimental task}

The task is illustrated in Fig. 1. Each trial of the task began with a $4 \mathrm{~mm}\left(\sim 0.4^{\circ}\right)$ fixation cross presented at the center of the computer screen. The time of the initial fixation interval varied randomly between 1600 and $2500 \mathrm{~ms}$. The fixation cross was continuously displayed during the whole trial. The target stimulus was an arrow pointing either left or right, displayed $18 \mathrm{~mm}\left(\sim 1.9^{\circ}\right)$ above or below the fixation cross (50/50) for $180 \mathrm{~ms}$. In each trial, the target arrow was flanked by four additional arrows (displayed simultaneously with the target) pointing to either the same or the opposite direction $(50 / 50)$, i.e., congruent or incongruent flankers respectively. The target and flanker arrows were each $9 \mathrm{~mm}\left(\sim 0.9^{\circ}\right)$ long. The total length of five arrows was $50 \mathrm{~mm}$ $\left(\sim 5.0^{\circ}\right)$. In half of the trials, a $2000 \mathrm{~Hz} 50 \mathrm{~ms}$ tone was presented as an alerting cue. The tone was presented $100 \mathrm{~ms}$ before the target, i.e., with $100 \mathrm{~ms}$ onset asynchrony (SOA). Participants were asked to respond to the target arrow by pressing the left or right Ctrl key on the computer keyboard with their left or right hand, respectively to the left- and rightpointing target arrow. Speed and accuracy of responses were measured. A new trial began automatically after the participant's response or after $2000 \mathrm{~ms}$ if the participant did not respond. All stimuli were black and were presented on a light gray background (RGB: 245,245,245). PsychoPy software (www.psychopy.org) was used for experimental control.

\subsection{Procedure}

At the beginning of the session, participants were given written and then verbal instructions describing the main task and the two SAT conditions. The speed instruction emphasized responding to the targets as quickly as possible while maintaining a reasonable level of accuracy. Participants were also explicitly informed that incorrect responses were invalid and would be removed from the analysis. The accuracy instruction emphasized responding as correctly as possible while not losing too much speed. The task began with a practice session in which 


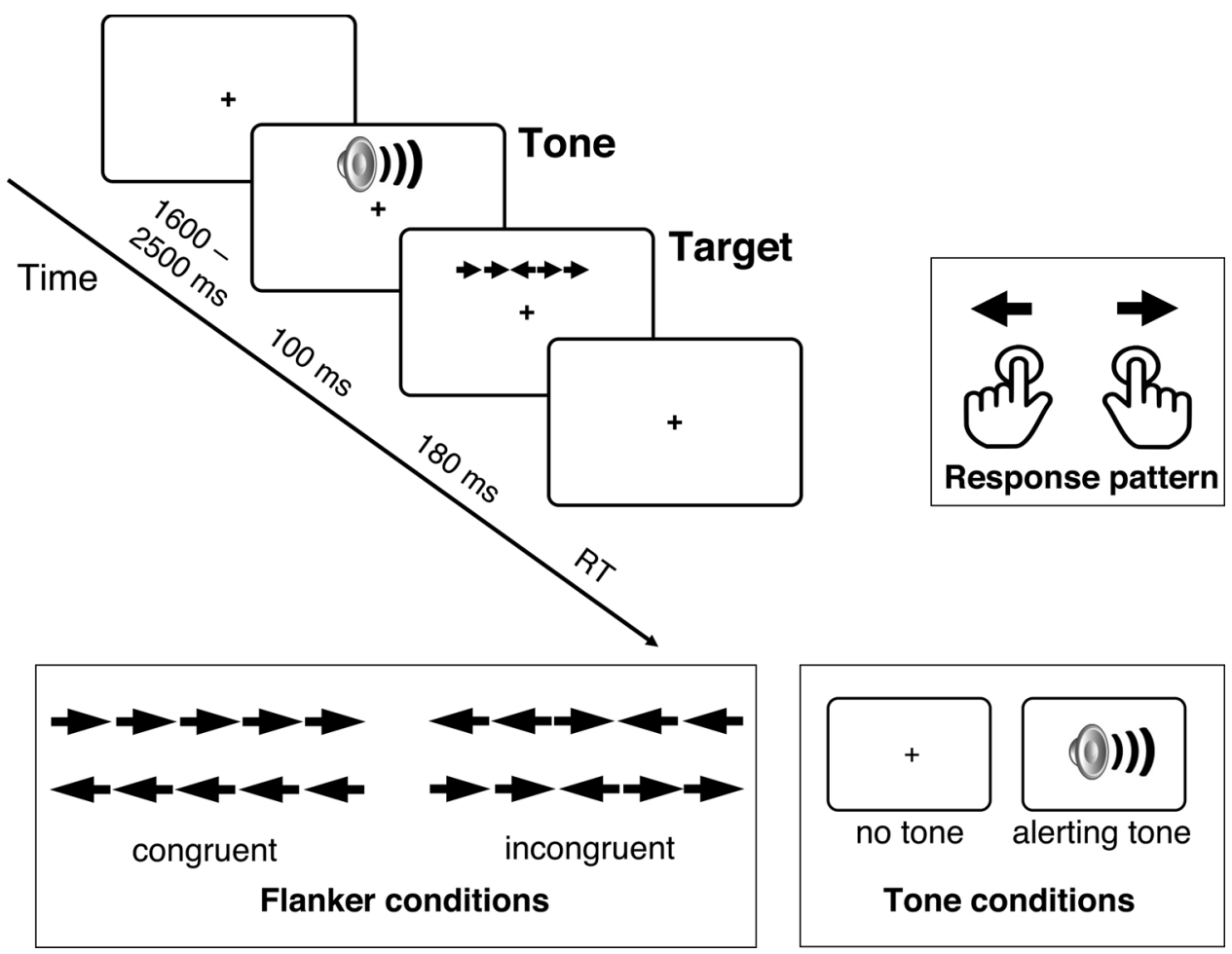

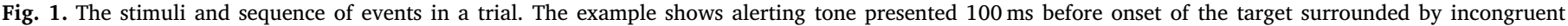
flankers. See Methods for details.

participants completed three blocks, each consisting of 16 trials. During the practice, participants received feedback on speed and accuracy after each response. The first practice block demonstrated the main task, and the next two blocks were focused on practicing the speed and accuracy strategies. After the practice session, participants were asked again whether they understood the task or had any question about the strategies. The practice session was followed by 640 experimental trials (with no feedback) divided into 10 blocks of 64 trials. In each block, the proportions of congruent/incongruent and tone/no-tone trials were 50/ 50 . The order of the alerting and flanker conditions was randomized within blocks individually for each participant. The speed and accuracy strategies were changed block-wise, with half of the participants beginning with the speed strategy and the other half with the accuracy strategy. A short remainder was displayed before each block, prompting participants to focus during the upcoming block on either speed or accuracy. Between the blocks, participants were asked to take breaks to rest their eyes. The task lasted up to one hour. The whole session lasted up to $90 \mathrm{~min}$.

\subsection{Behavioral data analysis}

Trials with incorrect response and trials with RT below or above 3 $S D$ were excluded from the RT analysis (overall 8.1\%). The RT and ERR data were submitted into $2 \times 2 \times 2$ repeated measure ANOVAs with Tone (tone, no-tone), Flanker (congruent, incongruent), and SAT (speed, accuracy) as within-subject factors.

\subsection{EEG data recording and preprocessing}

EEG was recorded using a BioSemi ActiveTwo system with $\mathrm{Ag}-\mathrm{AgCl}$ electrodes on 64 monopolar locations according to the extended 10-20 system, and two additional electrodes, the common mode sense (CMS) active electrode and the driven right leg (DRL) passive electrode, used as reference and ground electrodes, respectively (www.biosemi.com/ faq/cms\&drl.htm). All cephalic electrodes were placed on the scalp using the Electro-Cap. Vertical electrooculogram (EOG) was recorded from above and below the left eye and horizontal EOG was recorded from the external canthi of both eyes. The data was stored at a sampling rate of $256 \mathrm{~Hz}$.

Brain-Vision Analyzer software (version 2, Munich, Germany) was used for offline data processing. Data were filtered with a $0.016-40 \mathrm{~Hz}$ band-pass and $50 \mathrm{~Hz}$ band-rejection filters (Butterworth zero phase filters, attenuation of $12 \mathrm{~dB}$ /octave), re-referenced to linked mastoids, and split into appropriate segments for analysis of event-related potentials. Only segments from trials with correct responses were included. Segments were $1000 \mathrm{~ms}$ long, from $200 \mathrm{~ms}$ before target onset to $800 \mathrm{~ms}$ afterward.

Segmented data were referred to the first $100 \mathrm{~ms}$ of the segment as baseline, corrected for blinks and eye movement artifacts using the Gratton-Coles method (Gratton, Coles, \& Donchin, 1983) as implemented in the Brain-Vision Analyzer, and edited for other artifacts by rejecting trials with zero lines, overall minimum-maximum voltage differences $\geq 150 \mu \mathrm{V}$, voltage steps between adjacent data points $\geq 30$ $\mu \mathrm{V}$, and absolute amplitudes $\geq 100 \mu \mathrm{V}$. Artifact-free segments were averaged over each condition separately for each participant. The average of accepted segments per experimental condition was 65 $(\mathrm{SD}=13$, range $17-80)$.

\subsection{ERP measurement and analysis}

The N1 component of early visual potential evoked by target stimuli peaked over visual cortex of both hemispheres at about $200 \mathrm{~ms}$ after stimulus onset. The N1s formed fairly regular waveforms, well-defined at both hemispheres and easily identifiable in each participant. The components were measured in averages from the PO7 and PO8 sites, where the peaks were largest, using automatic peak detection within 140-260 ms after target onset. The peak latencies were determined relative to target onset. The amplitudes were determined relative to the preceding P1s (determined as the most positive peaks $80-140 \mathrm{~ms}$ after target onset) using a baseline-independent peak-to-peak method to 
bypass the issue of baseline affected by the tone-evoked activity in the tone trials (cf. Böckler et al., 2011). The N1 latencies and amplitudes were submitted to $2 \times 2 \times 2 \times 2$ repeated measure ANOVAs with within-subject factors of Tone (tone, no-tone), Flanker (congruent, incongruent), Hemisphere (PO7, PO8), and SAT (speed, accuracy).

The P3b component was measured in averages at the $\mathrm{Pz}$ site, where the peaks were largest, on data that were low-pass filtered at $5 \mathrm{~Hz}$. The latencies were measured using automatic peak detection at the most positive values $300-600 \mathrm{~ms}$ after target onset. The amplitudes were measured as mean voltage $300-600 \mathrm{~ms}$ after target onset relative to the negative N2-type component directly preceding the positive P3 peak (cf. Verleger, Śmigasiewicz, \& Möller, 2011), because in the tone trials, P3b always started at the positive side of the baseline (see Fig. 4A, red lines). The P3-baseline was measured separately for each participant as mean voltage 164-226 ms after target onset (the epoch specified based on the grand averages). This P3-baseline did not differ between the two Flanker conditions $(F<1.0$, n.s.).

The LRPs were calculated from activity recorded at C3 and C4, over the left and right motor cortex, as the average of contralateral-ipsilateral differences for the left-hand and right-hand correct responses, by the formula: ((C4 - C3) + (C3 - C4)) / 2 (Coles, 1989). Therefore, negative amplitude values reflect activation of the correct response, and positive amplitude values reflect activation of the incorrect response. The LRP onset latencies were estimated using the $50 \%$ of peak latency measure, which defines the onset latency as the time point at which the voltage reaches $50 \%$ of the peak amplitude (Kiesel, Miller, Jolicoeur, \& Brisson, 2008; Miller, Patterson, \& Ulrich, 1998). The amplitudes of the correct (negative) activation were estimated as mean activity $240-540 \mathrm{~ms}$ after target onset in the congruent trials and $380-680 \mathrm{~ms}$ after target onset in the incongruent trials. The initial incorrect (positive) activation, evoked by the incongruent flankers, formed well-defined peaks, which did not significantly differ in their latencies (see Results and Fig. 4B), hence their amplitudes were measured in a narrower time-window $260-320 \mathrm{~ms}$ after target onset. The JackKnife method was used for statistical analysis of the LRP latencies: the LRP parameters were measured in one-leave-out grand means, and the diminished error variance was corrected by dividing $F$ values by $(\mathrm{N}-1)^{2}$ (Kiesel et al., 2008; Ulrich \& Miller, 2001).

The ANOVA design in the P3b and LRP analyzes had the repeatedmeasurement factors Tone (tone, no-tone), Flanker (congruent, incongruent), and SAT (speed, accuracy), except for the analysis of the positive LRPs in the incongruent trials (absent in the congruent trials), which included only Tone and SAT factors.

Additionally, we analyzed amplitudes and latencies of the auditory N1 potential (cf. Pratt, 2011) evoked by alerting tone. The auditory N1 was measured at the FCz site, where it was largest, as the most negative peak 60-160 ms after tone onset. The latencies and amplitudes of the N1 potential were submitted to repeated measure ANOVAs with the factor SAT (speed, accuracy).

\subsection{Time-frequency calculation and analysis}

Time-frequency calculations were performed in Matlab (R2017a) using custom-written scripts based on Cohen (2014b, 2017). Single-trial EEG data were decomposed into a time-frequency representation by multiplying power spectrum via complex Morlet wavelet. Thirty frequencies ranging from 2 to $30 \mathrm{~Hz}$ were extracted from the single-trial EEG data using a logarithmically spaced number of cycles (steps), from 6 at $2 \mathrm{~Hz}$ to 10 at $30 \mathrm{~Hz}$. Frequency-band-specific power was estimated for each data time point from $-100 \mathrm{~ms}$ to $700 \mathrm{~ms}$ relative to target onset by squaring the magnitude of the result of the convolution between the wavelet and the EEG data $\left(\operatorname{real}[z(t)]^{2}+\operatorname{imag}[z(t)]^{2}\right)$. The obtained power values were normalized as a percentage change relative to the average pre-target baseline power at each frequency band (per subject, channel, condition, and epoch). The average time-window from $-200 \mathrm{~ms}$ to $-100 \mathrm{~ms}$ relative to target onset was used as the baseline.
Statistical analysis was focused on data from $\mathrm{FCz}$, based on inspection of topographical maps and a priori expectations based on previous studies. The precise time-frequency windows of conflict-related activity at the group level were identified by means of nonparametric tests, in which multiple comparisons were corrected at clusterlevel (Maris and Oostenveld, 2007). One thousand iterations of the permutation test were performed (across subjects). On each of the permutations, the conflict (incongruent-congruent) time-frequency map (in standard deviation units) was randomly shuffled, and a two-tailed $t$ test was performed on each time-frequency pixel against a null-distribution of $t$ values, providing a null-hypothesis distribution against which the observed results were compared at $p<0.001$. Clusters in the real data that were equal or larger than $99 \%$ of this distribution of null hypothesis cluster sizes were considered statistically significant.

Conflict-related oscillatory activity was found, as expected, within the window of $4-8 \mathrm{~Hz}$ and $400-600 \mathrm{~ms}$ after target onset. To test the effects of experimental manipulations, average theta power of this timefrequency window was computed for each of the eight conditions per participant and submitted into a $2 \times 2 \times 2$ repeated measure ANOVA with Tone (tone, no-tone), Flanker (congruent, incongruent), and SAT (speed, accuracy) as within-subject factors. For brevity, only relevant effects in terms of conflict-related theta activity are reported.

\section{Results}

\subsection{Behavioral results}

The overall mean RT was $548 \mathrm{~ms}(S D=83 \mathrm{~ms})$ and the overall mean ERR was 7\% $(S D=4 \%)$. Mean RT and ERR for each condition are shown in Table 1 and Fig. 2 (upper panels). Indices of the conflict effect (incongruent minus congruent flanker condition) for each Tone and SAT condition are shown in Fig. 2 (lower panels).

\subsubsection{Main effects}

All three main effects were significant in both RT and ERR measurements. The effect of Flanker (conflict effect) showed that responses were faster and more accurate in the congruent than in the incongruent condition: $494 \mathrm{~ms}$ vs. $602 \mathrm{~ms}, F_{1,26}=369.55, p<.001, \eta_{p}{ }^{2}=.93$, and $1 \%$ vs. $14 \%, F_{1,26}=75.52, p<.001, \eta_{p}{ }^{2}=.74$. The effect of Tone (alerting effect) showed that responses were faster but less accurate in the trials with tone than in the no-tone trials: $531 \mathrm{~ms}$ vs. $564 \mathrm{~ms}$, $F_{1,26}=204.04, p<.001, \eta_{p}{ }^{2}=.89$, and $9 \%$ vs. $6 \%, F_{1,26}=8.95, p=$ $.006, \eta_{p}{ }^{2}=.26$. Finally, the effect of SAT showed, as expected, that speed strategy resulted in generally faster but less accurate responses than the accuracy strategy, showing typical effect of speed-accuracy tradeoff: $525 \mathrm{~ms}$ vs. $570 \mathrm{~ms}, F_{1,26}=45.63, p<.001, \eta_{p}{ }^{2}=.64$, and $10 \%$ vs. $5 \%, F_{1,26}=26.12, p<.001, \eta_{p}{ }^{2}=.50$.

\subsubsection{Interactions}

In RTs, the flanker effect was larger in the tone trials $(118 \mathrm{~ms})$ than in the no-tone trials $(97 \mathrm{~ms})$. Specifically, the Tone $\times$ Flanker

\section{Table 1}

The average response time of correct responses (RT) and the average error rate (ERR) for each experimental condition (the values in brackets are standard deviations).

\begin{tabular}{lllll}
\hline Alerting condition & \multirow{2}{*}{ SAT condition } & Flanker type & RT (ms) & ERR (\%) \\
\hline no-tone & speed & congruent & $494(50)$ & $1.0(1.0)$ \\
& & incongruent & $589(50)$ & $14.0(10.1)$ \\
& accuracy & congruent & $536(56)$ & $0.5(0.9)$ \\
tone & & incongruent & $636(65)$ & $8.1(6.3)$ \\
& speed & congruent & $450(46)$ & $1.2(1.5)$ \\
& & incongruent & $567(53)$ & $22.3(14.7)$ \\
& accuracy & congruent & $495(54)$ & $0.4(0.7)$ \\
& & incongruent & $614(74)$ & $10.0(8.1)$ \\
\hline
\end{tabular}



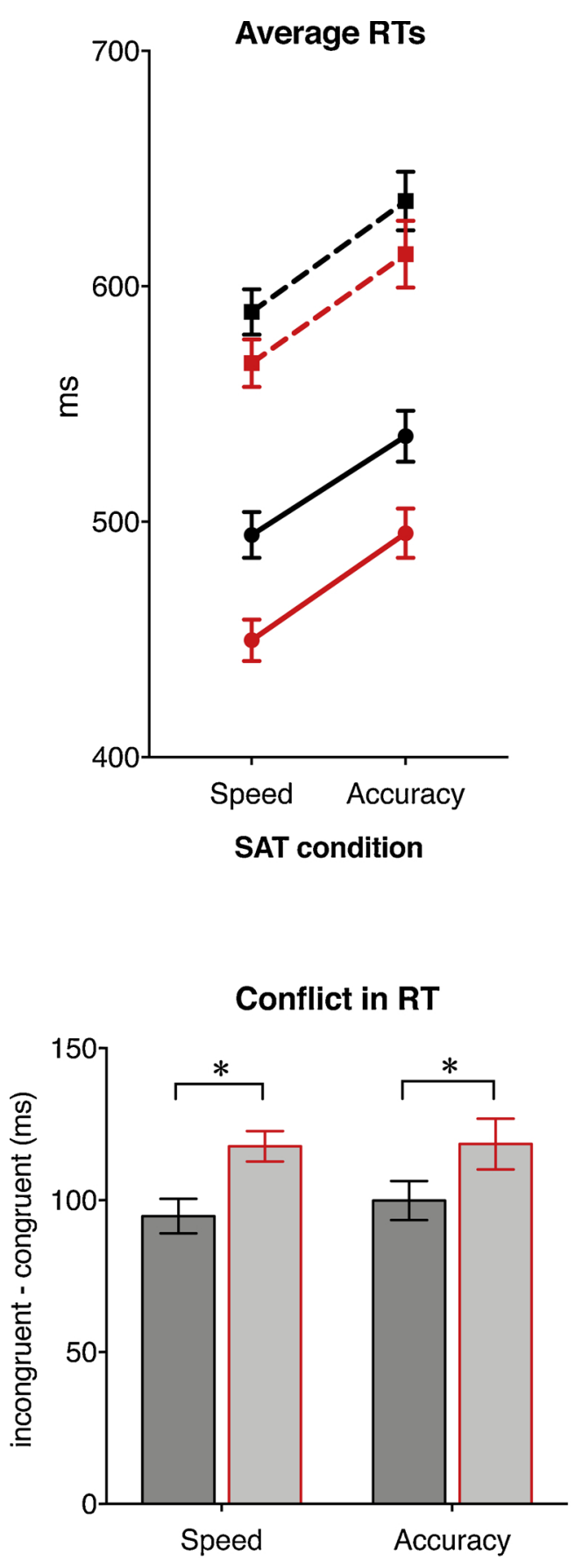

SAT condition

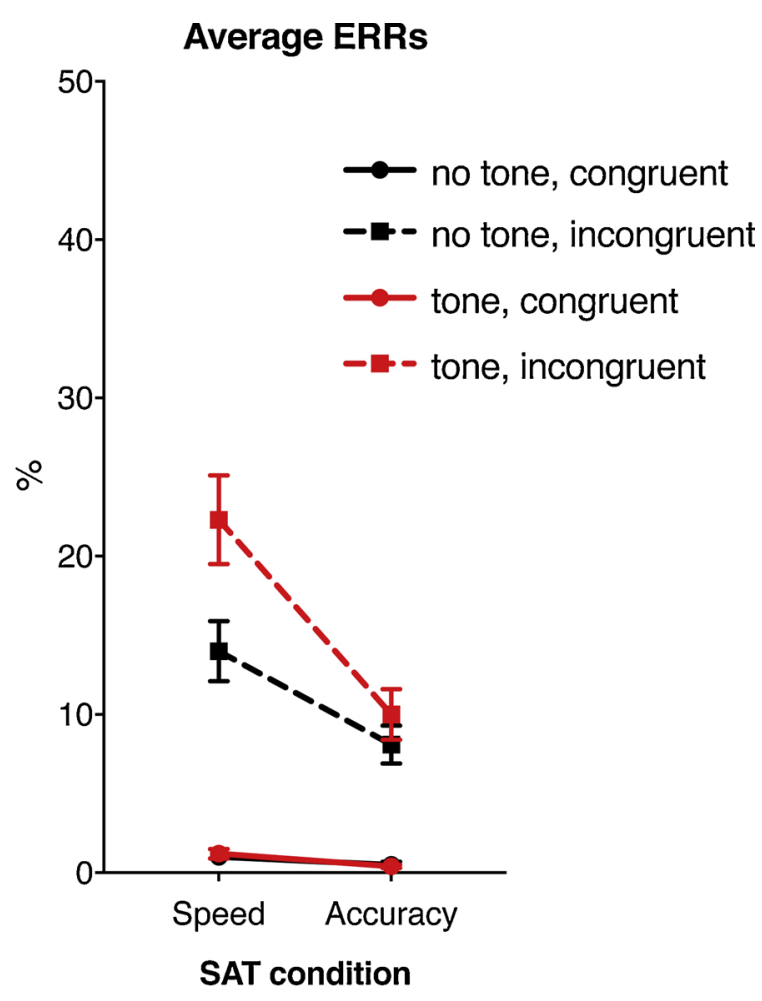

Conflict in ERR

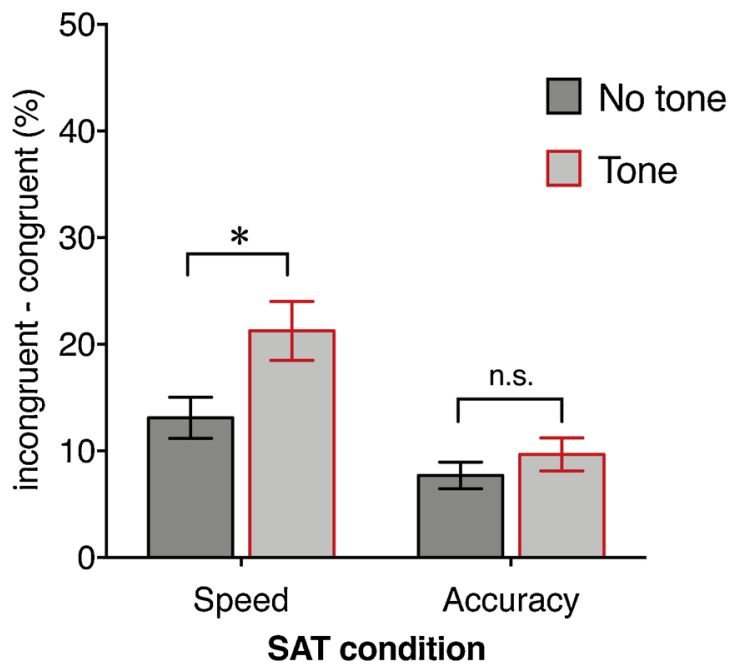

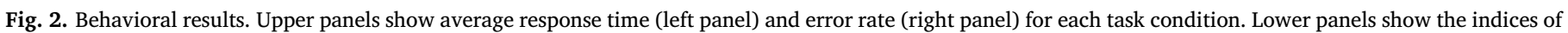
flanker conflict effect (incongruent-congruent flankers) calculated from the RTs (left panel) and ERRs (right panel). Error bars represent standard errors.

interaction, $F_{1,26}=21.85, p<.001, \eta_{p}{ }^{2}=.46$, showed that the effect of decreased RT in the tone trials (i.e., the "standard" alerting effect) was larger in the congruent than in the incongruent condition (effect of Tone in the congruent condition, $515 \mathrm{~ms}$ vs. $472 \mathrm{~ms}, F_{1,26}=281.69$, $p<.001, \eta_{p}{ }^{2}=.91$; effect of Tone in the incongruent condition, $612 \mathrm{~ms}$ vs. $590 \mathrm{~ms}: F_{1,26}=35.70, p<.001, \eta_{p}{ }^{2}=.58$ ). Of importance, this Tone $\times$ Flanker interaction in RT was independent of SAT manipulation (SAT $\times$ Tone $\times$ Flanker interaction in RT: $F<1.0$, n.s.). Furthermore, in RT, the main effects of Tone and SAT were additive, not interactive, as indicated by the non-significant Tone $\times$ SAT interaction, $F<1.0$, n.s. Finally, SAT manipulation did not affect the flanker effect in RT, SAT $\times$ Flanker: $F<1.0$, n.s.
In ERR measurement, the Tone $\times$ Flanker interaction was significant, $F_{1,26}=9.00, p=.006, \eta_{p}{ }^{2}=.26$, as in the RTs, but so was the Tone $\times$ SAT, $\quad F_{1,26}=19.85, \quad p<.001, \quad \eta_{p}{ }^{2}=.43$, and the Tone $\times$ Flanker $\times$ SAT, $F_{1,26}=16.55, p<.001, \eta_{p}{ }^{2}=.39$, indicating that the effect of Tone and the relationship between Tone and Flanker was modulated by SAT. When resolving this three-way interaction, significant effect of Tone and significant Tone $\times$ Flanker interaction were found in the speed condition, but not in the accuracy condition. In detail, in the speed condition, the effect of Flanker was larger in the tone trials than in the no-tone trials (Tone $\times$ Flanker: $F_{1,26}=15.58$, $p=.001, \eta_{p}{ }^{2}=.37$ ); the congruent vs. incongruent difference in the tone trials was $21 \%, F_{1,26}=58.43, p<.001, \eta_{p}{ }^{2}=.69$, while in the 
Table 2

Estimated latencies and amplitudes of the N1, P3b, and LRP components of the ERPs in each relevant experimental condition. See Method (Section 2.6) for details.

\begin{tabular}{|c|c|c|c|c|c|}
\hline \multicolumn{6}{|l|}{ Visual N1 } \\
\hline $\begin{array}{l}\text { Alerting } \\
\text { condition }\end{array}$ & SAT & Hemisphere & Flanker type & Latency (ms) & Amplitude $(\mu \mathrm{V})$ \\
\hline \multirow[t]{8}{*}{ no-tone } & \multirow[t]{4}{*}{ speed } & \multirow[t]{2}{*}{ PO7 } & congruent & 197 & $-8,7$ \\
\hline & & & incongruent & 199 & $-9,1$ \\
\hline & & \multirow[t]{2}{*}{ PO8 } & congruent & 192 & $-8,6$ \\
\hline & & & incongruent & 195 & $-9,0$ \\
\hline & \multirow[t]{4}{*}{ accuracy } & \multirow[t]{2}{*}{ PO7 } & congruent & 197 & $-8,3$ \\
\hline & & & incongruent & 196 & $-8,9$ \\
\hline & & \multirow[t]{2}{*}{ PO8 } & congruent & 192 & $-8,3$ \\
\hline & & & incongruent & 196 & $-8,5$ \\
\hline \multirow[t]{8}{*}{ tone } & \multirow[t]{4}{*}{ speed } & \multirow[t]{2}{*}{ PO7 } & congruent & 182 & $-7,1$ \\
\hline & & & incongruent & 184 & $-7,0$ \\
\hline & & \multirow[t]{2}{*}{ PO8 } & congruent & 182 & $-7,2$ \\
\hline & & & incongruent & 183 & $-7,4$ \\
\hline & \multirow[t]{4}{*}{ accuracy } & \multirow[t]{2}{*}{ PO7 } & congruent & 186 & $-7,2$ \\
\hline & & & incongruent & 183 & $-7,4$ \\
\hline & & \multirow[t]{2}{*}{ PO8 } & congruent & 183 & $-7,3$ \\
\hline & & & incongruent & 182 & $-7,5$ \\
\hline
\end{tabular}

\begin{tabular}{|c|c|c|c|c|}
\hline Alerting condition & SAT & Flanker type & Latency (ms) & Amplitude $(\mu \mathrm{V})$ \\
\hline \multirow[t]{4}{*}{ no-tone } & speed & congruent & 464 & 13,7 \\
\hline & & incongruent & 479 & 11,4 \\
\hline & accuracy & congruent & 446 & 11,6 \\
\hline & & incongruent & 489 & 10,4 \\
\hline \multirow[t]{4}{*}{ tone } & speed & congruent & 436 & 8,4 \\
\hline & & incongruent & 480 & 7,3 \\
\hline & accuracy & congruent & 398 & 7,2 \\
\hline & & incongruent & 471 & 6,7 \\
\hline
\end{tabular}

\begin{tabular}{|c|c|c|c|c|}
\hline LRP & & & & \\
\hline Alerting condition & SAT & $\begin{array}{l}\text { Flanker } \\
\text { type }\end{array}$ & Onset latency (ms) & Amplitude $(\mu \mathrm{V})$ \\
\hline \multirow[t]{4}{*}{ no-tone } & speed & congruent & 284 & $-1,7$ \\
\hline & & incongruent & 411 & $-1,4$ \\
\hline & accuracy & congruent & 274 & $-1,3$ \\
\hline & & incongruent & 397 & $-1,5$ \\
\hline \multirow[t]{4}{*}{ tone } & speed & congruent & 267 & $-1,5$ \\
\hline & & incongruent & 383 & $-2,2$ \\
\hline & accuracy & congruent & 275 & $-1,3$ \\
\hline & & incongruent & 392 & $-0,9$ \\
\hline \multicolumn{5}{|l|}{ positive LRP } \\
\hline Alerting condition & \multicolumn{2}{|c|}{ SAT } & Latency (ms) & Amplitude $(\mu \mathrm{V})$ \\
\hline \multirow[t]{2}{*}{ no-tone } & \multicolumn{2}{|c|}{ speed } & 304 & 0,9 \\
\hline & \multicolumn{2}{|c|}{ accuracy } & 300 & 0,7 \\
\hline \multirow[t]{2}{*}{ tone } & \multirow{2}{*}{\multicolumn{2}{|c|}{$\begin{array}{l}\text { speed } \\
\text { accuracy }\end{array}$}} & 293 & 1,2 \\
\hline & & & 305 & 1,5 \\
\hline
\end{tabular}

no-tone trials it was only $13 \%, F_{1,26}=44.93, p<.001, \eta_{p}{ }^{2}=.63$ (the overall tone effect in the speed trials was $4 \%, F_{1,26}=17.00, p<.001$, $\eta_{p}^{2}=.39$ ). In the accuracy condition, however, both the effect of Tone and the Tone $\times$ Flanker interaction were not significant, $F_{1,26}=1.16$, $p=.29, \eta_{p}{ }^{2}=.04$, and $F_{1,26}=1.51, p=.23, \eta_{p}{ }^{2}=.05$, respectively. The overall average of the Flanker effect in the accuracy condition was $9 \%, F_{1,26}=57.41, p<.001, \eta_{p}^{2}=.69$.

\subsection{ERP results}

Estimations of mean ERP latencies and amplitudes for each relevant experimental condition are shown in Table 2.

\subsubsection{Tone-evoked anterior auditory $N 1$}

Alerting tone evoked a conspicuous negative potential, typical for auditory stimuli, which peaked at FCz about $100 \mathrm{~ms}$ after tone onset. Neither the amplitude nor latency of this auditory N1 was affected by SAT manipulation ( $F \mathrm{~s}<1.0$, n.s.).

\subsubsection{Target-evoked posterior visual N1}

Latency. The visual $\mathrm{N} 1$ peaked in average about $12 \mathrm{~ms}$ earlier in the tone trials than in the no-tone trials ( $183 \mathrm{~ms}$ vs. $195 \mathrm{~ms}), F_{1,26}=71.35$, $p<.001, \eta_{p}{ }^{2}=.73$ (see Fig. 3). No other main effects or interactions were significant, $F \leq 1.5, p \geq .23$.

Amplitude. The N1-P1 amplitude difference was smaller in the tone trials than in the no-tone trials, $F_{1,26}=10.24, p=.004, \eta_{p}{ }^{2}=.28$ (Fig. 3). There was a trend for smaller amplitude in congruent than in the incongruent flanker condition, $F_{1,26}=3.42, p=.076, \eta_{p}{ }^{2}=.12$. There was also a small trend for larger amplitudes in the no-tone speed trials (see Table 2), but the Tone $\times$ SAT interaction was not significant, $F_{1,26}=3.14, p=.09, \eta_{p}{ }^{2}=.11$; nor were the other effects, $F \leq 1.8$, $p \geq .19$.

\subsubsection{Target-evoked $P 3 b$}

As seen in Fig. 4A, the P3b components in the tone trials had notably shorter latencies and smaller amplitudes than in the no-tone trials (red vs. black lines), while the P3bs evoked by the targets with incongruent flankers had smaller and more prolonged, two-phase-like waveforms (with largest amplitudes at the later phase), compared to the targets with congruent flankers (dashed vs. solid lines). The peak topographies were however very similar in both the congruent and incongruent conditions. Of importance, this flanker effect on P3b was notably modified by alerting tone. Finally, there was a small increase in the P3b amplitudes in the speed condition, compared to the accuracy condition (Fig. 5, thin vs. bold lines).

Latency. Statistical analysis confirmed that P3b peaked significantly earlier in the tone trials than in the no-tone trials, $F_{1,26}=5.74, p=$ $.024, \eta_{p}{ }^{2}=.18$. Also, the main effect of Flanker was significant: P3b peaks were significantly delayed in the incongruent trials, compared to the congruent trials, $F_{1,26}=9.62, p=.005, \eta_{p}{ }^{2}=.27$. This conflict effect (i.e., the delay of the P3b latency) tended to be larger in the tone trials, as indicated by a marginally significant Tone $\times$ Flanker interaction, $F_{1,26}=3.68, p=.066, \eta_{p}{ }^{2}=.12$. The main effect of SAT was not significant, $F_{1,26}=2.02, p=.17, \eta_{p}^{2}=.07$, nor were the other interactions, $F \leq 1.8, p \geq .19$.

Amplitude. All three main effects were significant. The P3b amplitudes were larger in the no-tone than in the tone trials, $F_{1,26}=25.52$, $p<.001, \eta_{p}{ }^{2}=.49$ (Fig. 4A, black vs. red lines); were larger in the congruent than in the incongruent trials, $F_{1,26}=19.18, p<.001$, $\eta_{p}{ }^{2}=.42$ (Fig. 4A, solid vs. dashed lines); and were larger in the speed than in the accuracy condition, $F_{1,26}=27.76, p<.001, \eta_{p}{ }^{2}=.52$ (Fig. 5, thin vs. bold lines).

The flanker conflict effect was modulated independently by Tone and by SAT, as indicated by significant interactions Tone $\times$ Flanker, $F_{1,26}=5.74, p=.024, \eta_{p}{ }^{2}=.18$ (Fig. 4A), and SAT $\times$ Flanker, $F_{1,26}=6.28, p=.019, \eta_{p}^{2}=.19$ (Fig. 5), and a non-significant threeway Tone $\times$ Flanker $\times$ SAT interaction, $\quad F_{1,26}=1.57, \quad p=.22$, $\eta_{p}{ }^{2}=.06$. Specifically, the flanker effect was much smaller (and only marginally significant) in the tone trials, $F_{1,26}=3.50, p=.073$, $\eta_{p}{ }^{2}=.12$, compared to the no-tone trials, $F_{1,26}=44.03, p<.001$, $\eta_{p}{ }^{2}=.63$, predominantly due to decreased amplitudes in the tone congruent trials, compared to the no-tone congruent trials. Further, the flanker effect was larger in the speed condition, $F_{1,26}=32.39, p<$ $.001, \eta_{p}{ }^{2}=.55$, than in the accuracy condition, $F_{1,26}=5.36, p=.029$, $\eta_{p}{ }^{2}=.17$. The Tone $\times$ SAT interaction was not significant, $F_{1,26}=3.14, p=.09, \eta_{p}^{2}=.11$.

\subsubsection{Lateral readiness potential (LRP)}

Fig. 4B shows grand means of the LRPs recorded at C3/C4 for the 


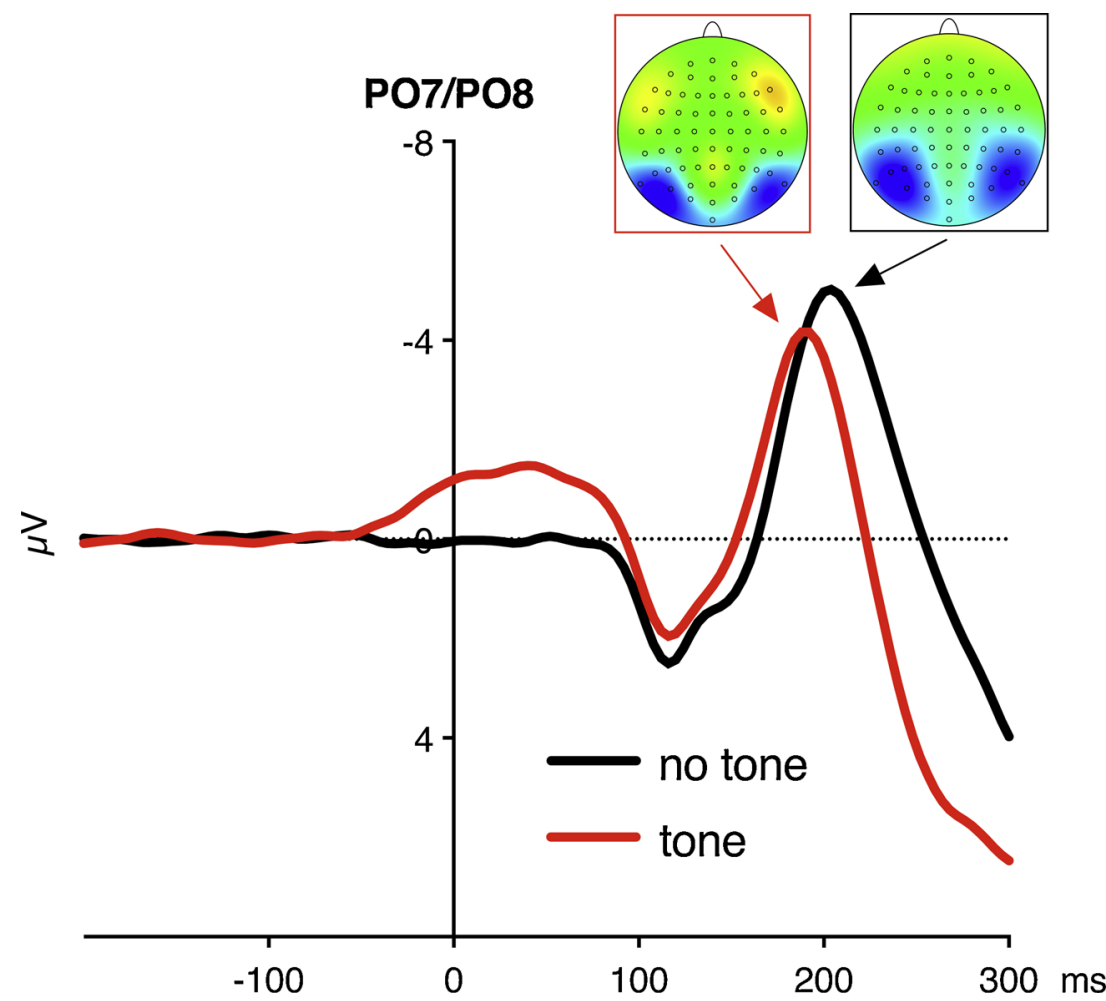

Fig. 3. Grand means of early visual evoked potentials in the two tone conditions (red line: tone trials, black line: no-tone trials) pooled across the two flanker and two SAT conditions, and PO7 and PO8 sites. Negative voltage points upwards. Time-point zero is onset of target and flankers. The head maps depict topographies of the N1 maxima. The maps are min-max scaled, with positive polarity in red, negative polarity in blue. The head view is from above. Colors of arrows and frames denote the experimental conditions.

four Tone $\times$ Flanker combinations. In the congruent trials, the LRP diverged from zero at about $240 \mathrm{~ms}$ after target onset and formed negative waves reflecting activation of the correct response. In the incongruent trials, the LRPs began to diverge from zero at about same time as in the congruent trials, but in the opposite, positive direction, reflecting initial activation of the incorrect response triggered by the incongruent flankers. This incorrect activation was larger in the tone trials than in the no-tone trials (red vs. black lines). The positive deflection reversed its polarity after about $140 \mathrm{~ms}$ and eventually also formed negative waves corresponding with correct but delayed behavioral responses. A trend for faster onsets of the negative LRP peaks in the tone trials than in the no-tone trials (red vs. black lines) is noticeable in both the congruent and incongruent conditions (solid and dashed lines).

Latency. The onset latency of the negative (correct) deflection was about $122 \mathrm{~ms}$ longer in the incongruent condition $(396 \mathrm{~ms})$ than in the congruent flanker condition (276 ms), $F_{1,26}=290.00, p<.001$, $\eta_{p}{ }^{2}=.92$, which closely resembles the present flanker effect in RTs. No other effects were significant, $F \leq 2.50, p \geq .13$, except a marginally significant main effect of Tone, reflecting the aforementioned trend for quicker LRP onsets in the tone trials ( $329 \mathrm{~ms}$ ) than in the no-tone trials (342 ms), $F_{1,26}=3.47, p=.07, \eta_{p}^{2}=.12$.

No significant differences were found in the latencies of the initial positive (incorrect) activation, $F$ s $<1.0$.

Amplitude. Importantly, the initial incorrect positive potential evoked by the incongruent flankers was significantly larger in the trials with tone than in the no-tone trials, $F_{1,26}=5.48, p=.027, \eta_{p}{ }^{2}=.17$, reflecting stronger activation of the incorrect response. This result corresponds with the present RT and ERR results showing larger flanker conflict in the tone trials. The Tone effect tended to be larger in the accuracy condition than in the speed condition, but the Tone $\times$ SAT interaction did not reach the significance level, $F_{1,26}=2.16, p=.15$, $\eta_{p}{ }^{2}=.08$. The main effect of SAT also was not significant, $F<1.0$.

The effects of Tone, Flanker, and Tone $\times$ Flanker on the negative (correct) LRP amplitudes were not significant $(F \mathrm{~s}<1.0)$, whereas the effect of SAT was significant, indicating larger LRP amplitude in the speed condition than in the accuracy condition, $F_{1,26}=8.13, p=.008$, $\eta_{p}{ }^{2}=.24$. This SAT effect tended to be larger in the tone trials (Tone $\times$ SAT: $F_{1,26}=3.86, p=.06, \eta_{p}{ }^{2}=.13$; averages for each condition are presented in Table 2).

\subsection{Time-frequency results}

Power estimations of the theta-band conflict effect (incongruentcongruent), measured at $\mathrm{FCz}$ in the $4-8 \mathrm{~Hz}$ frequency range, 400-600 ms after target onset, are depicted in Table 3 and Fig. 4C and 4D. The midfrontal theta power was conspicuously enhanced in the conflict trials, compared to the no-conflict trials, and peaked at about 6-7 Hz, 450-500 ms after target onset. Crucially, as seen in Fig. 4C and $\mathrm{D}$, this conflict-related theta power was larger in the tone trials than in the no-tone trials. The ANOVA confirmed these findings, showing a significant main effect of Flanker, $F_{1,26}=23.85, p<.001, \eta_{p}{ }^{2}=.48$, and a significant Tone $\times$ Flanker interaction, $F_{1,26}=10.56, p=.003$, $\eta_{p}{ }^{2}=.29$. The permutation tests showed that conflict-related theta power was significant at $p<.001$ (two-tailed, cluster corrected) at about $4-9 \mathrm{~Hz}, 300-600 \mathrm{~ms}$ after target onset in the tone trials, and 5-8 Hz, 380-600 ms in the no-tone trials (see Fig. 4C).

Although there was a trend for a larger conflict theta effect in the speed condition than in the accuracy condition, the ANOVA showed no significant SAT effects on the conflict-related theta activity (Flanker $\times$ SAT: $\quad F_{1,26}=2.47, \quad p=\quad .13, \quad \eta_{p}{ }^{2}=.09$; Tone $\times$ Flanker $\times$ SAT: $F<1.0$.)

\section{Discussion}

\subsection{Effects of alerting on conflict}

\subsubsection{Summary of results}

The behavioral results showed the usual effect of phasic alerting on response conflict: The conflict was larger in the trials with alerting tone than in the no-tone trials, which replicates the findings of earlier studies (Asanowicz \& Marzecová, 2017; Böckler et al., 2011; Callejas et al., 2004; Fischer et al., 2010; Ishigami \& Klein, 2010; Weinbach \& Henik, 2013). 
In the ERP measurement, this alerting-conflict interaction was reflected in the modulations of two components: P3b and LRP. The P3b latencies were longer and the P3b amplitudes were smaller in the incongruent condition than in the congruent condition, which replicates the previous findings (Kałamała et al., 2017, 2018; Neuhaus et al., 2010; Osman et al., 2000; Smid et al., 1990). This result conforms to our presumption that response conflict delays the $S-R$ translation and activation of the correct S-R link (as reflected in P3b, see the

\section{Tone $\times$ Flanker}

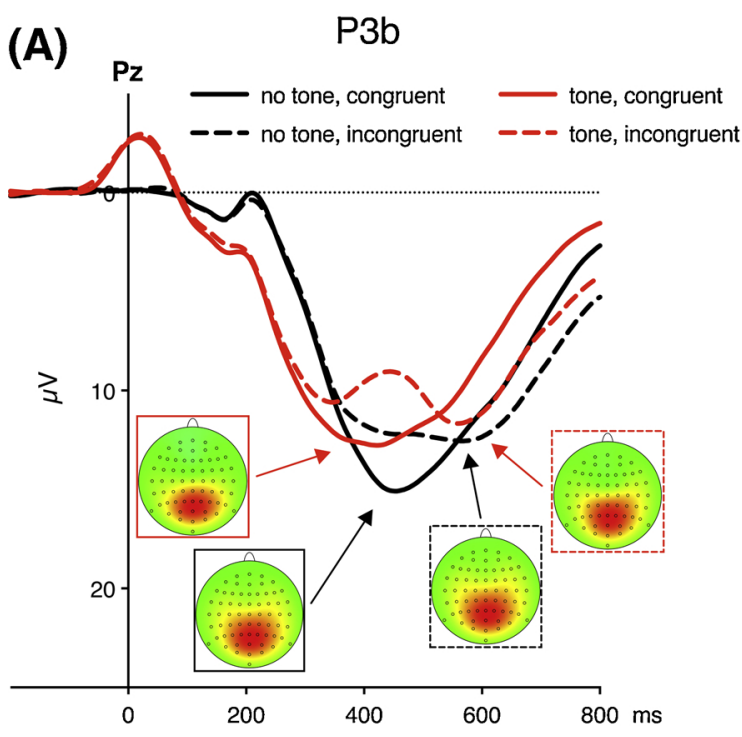

(B)

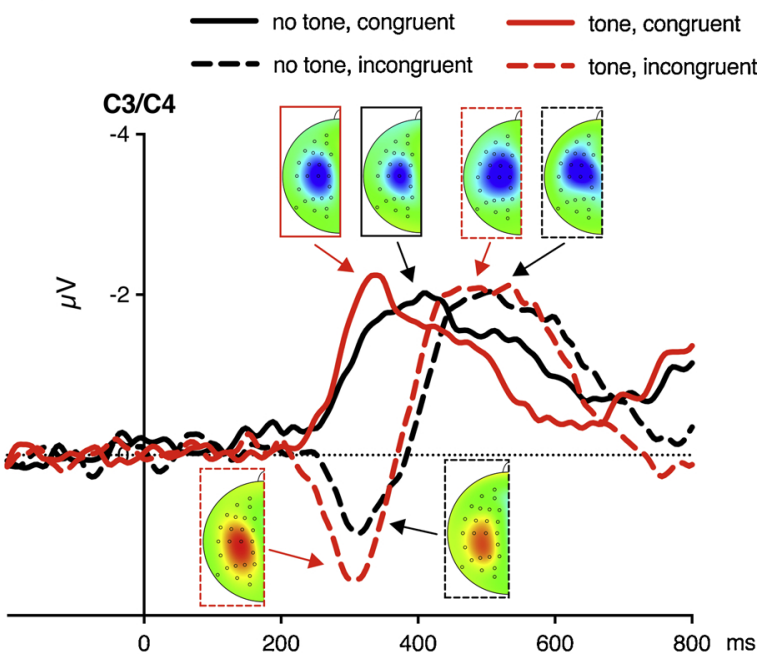

(C)
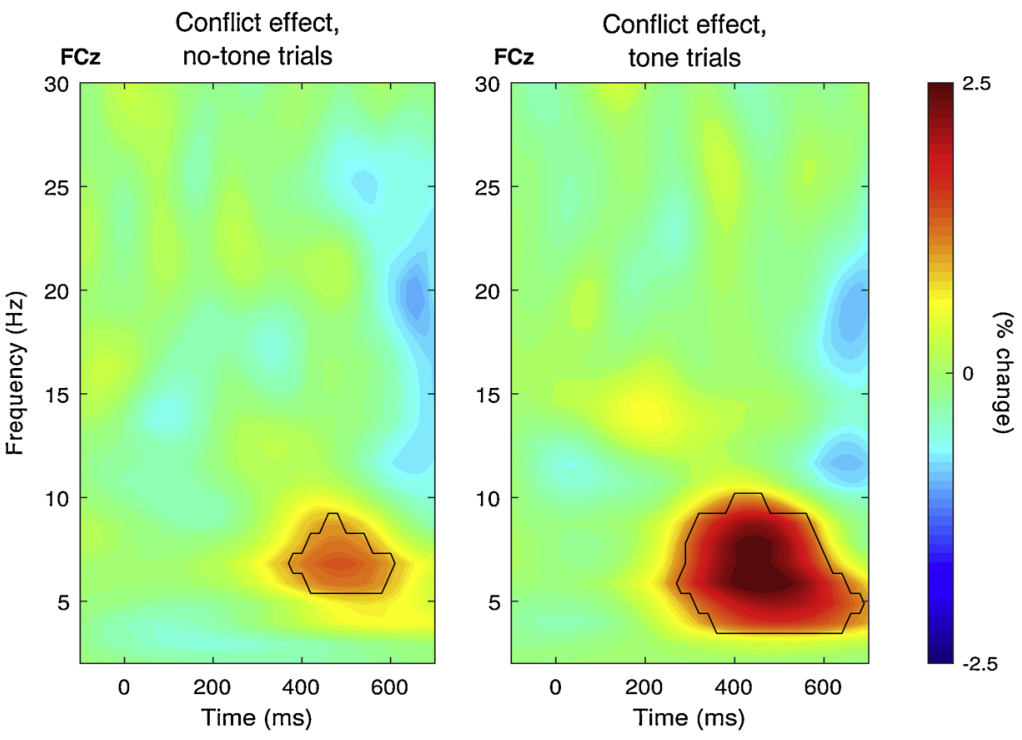

(D)
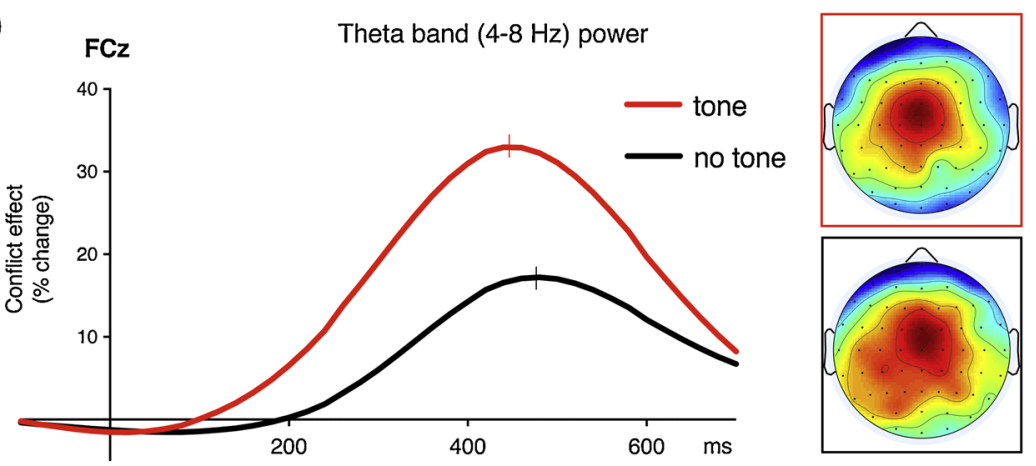


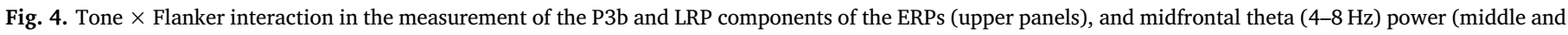
lower panels).

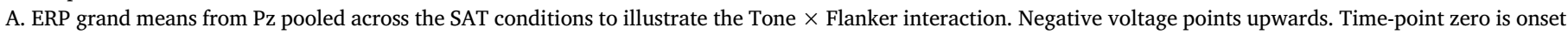

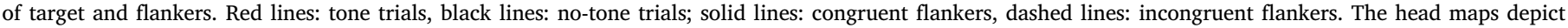

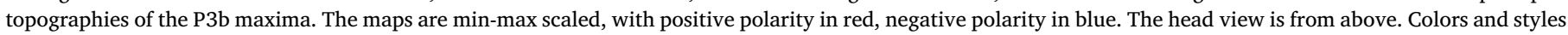
of frames denote the conditions.

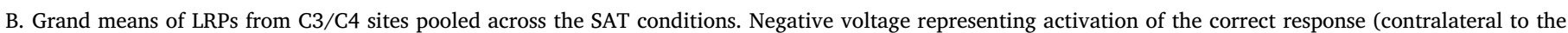

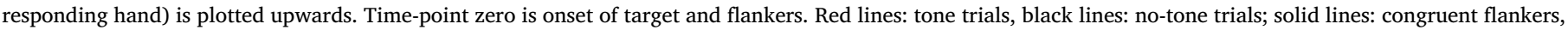

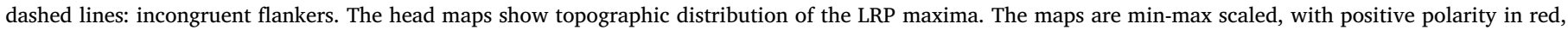
negative polarity in blue. The head view is from above. Colors and styles of frames denote the conditions.

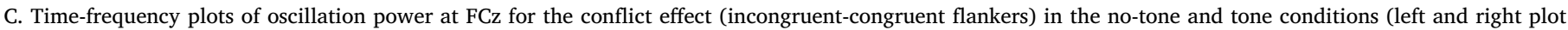

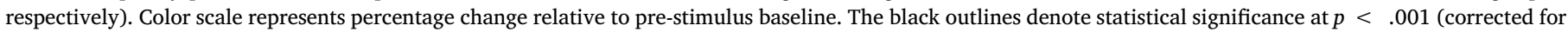

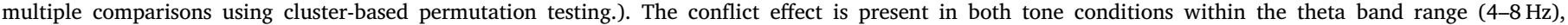
400-600 ms after target onset.

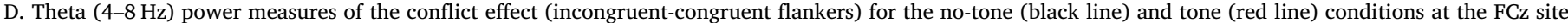

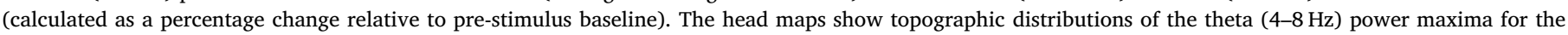

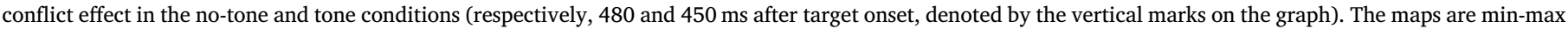
scaled.

introduction). Importantly, these deteriorating effects of incongruent flankers on P3b were enhanced by alerting: In the conflict trials with alerting stimulus, the P3b amplitudes were even smaller and the P3b latencies tended to be even longer than in the conflict trials without alerting. Furthermore, the LRP results showed that alerting increased the amplitude of the initial activation of the incorrect response, whereas neither the amplitude of the subsequently emerged correct activation nor the LRP amplitude in the congruent condition were significantly affected by alerting. These LRP results suggest that alerting increases the conflict specifically by enhancing the activation of the incorrect response evoked by the incongruent flankers. As mentioned in the introduction, this erroneous S-R link (incongruent flankers $\rightarrow$ incorrect response) is thought to be triggered automatically via a direct, reflex-like S-R route. The present results suggest that phasic alerting facilities this automatic S-R translation.

Furthermore, the ERP results showed that alerting facilitated and increased the N1 component of visual-evoked potential, corroborating previous findings of the impact of alerting on visual processing (Böckler

\section{Flanker $\times$ SAT}

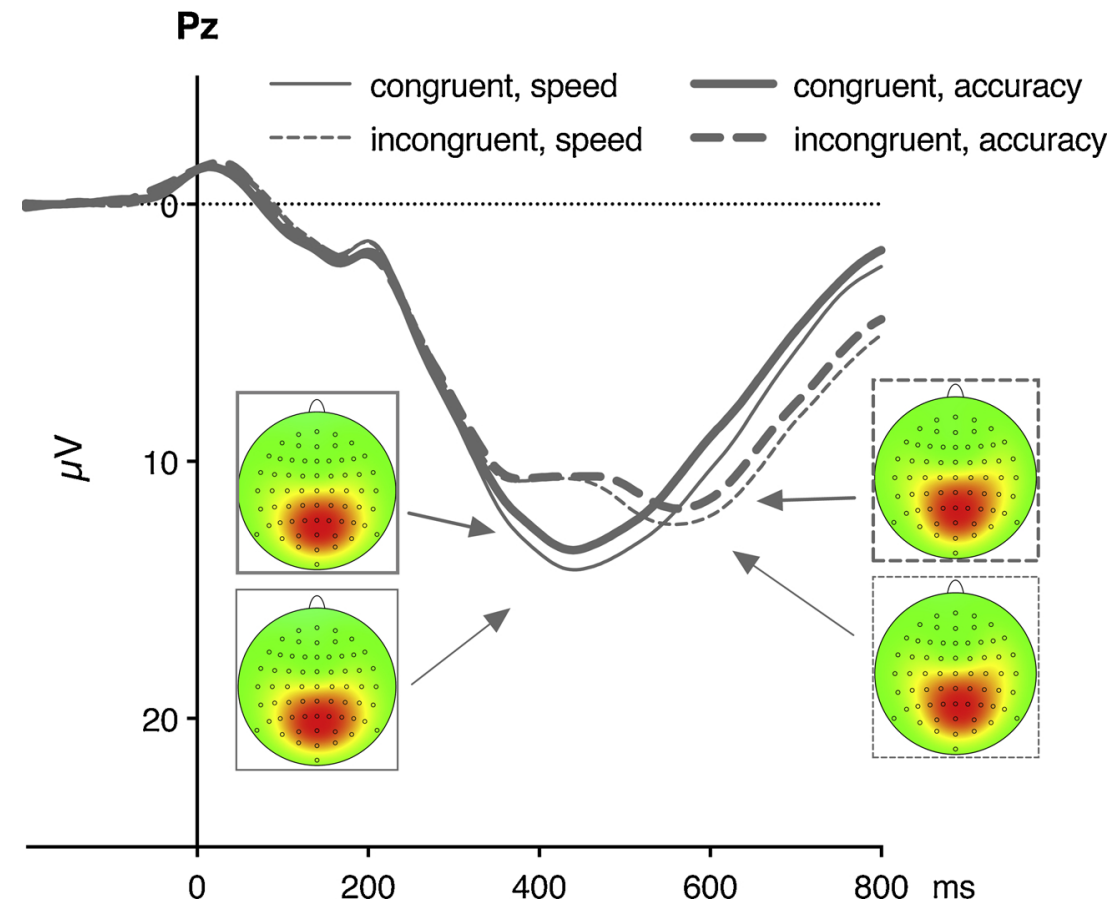

Table 3

Power estimations of the theta-band conflict effect (incongruent-congruent) measured at FCz in the $400-600 \mathrm{~ms}, 4-8 \mathrm{~Hz}$ windows. See Method (Section 2.7) for details.

\begin{tabular}{lll}
\hline Alerting condition & SAT condition & $\begin{array}{l}\text { Conflict effect } \\
\text { (\% change) }\end{array}$ \\
\hline no-tone & speed & 18 \\
\multirow{2}{*}{ tone } & accuracy & 14 \\
& speed & 33 \\
& accuracy & 24 \\
\hline
\end{tabular}

et al., 2011; Jepma, Wagenmakers, Band, \& Nieuwenhuis, 2009), and also, that alerting tended to accelerate onsets of the correct response activation in LRPs, again in line with earlier reports (Böckler et al., 2011; Hackley \& Valle-Inclán, 1998; Jepma et al., 2009). These two effects, however, were found to be unaffected by the flanker manipulation, which suggests that they are not related to the alerting-conflict interaction.

Fig. 5. ERP grand means from $\mathrm{Pz}$ pooled across the tone conditions to illustrate the SAT $\times$ Flanker interaction. Negative voltage points upwards. Time-point zero is onset of target and flankers. Solid lines: congruent flankers, dashed lines: incongruent flankers; thin lines: speed condition, thick lines: accuracy condition. The head maps depict topographies of the P3b maxima. The maps are min-max scaled, with positive polarity in red and negative polarity in blue. The head view is from above. Colors and styles of frames denote the experimental conditions. 
The time-frequency results showed an enhanced midfrontal thetaband activity in the conflict trials, compared to the no-conflict trials, which is thought to reflect the oscillatory mechanism of conflict processing (Cavanagh \& Frank, 2014; Cohen, 2014a). This result replicates the findings of other studies using the flanker task (Cavanagh et al., 2009; Cohen \& Cavanagh, 2011; Nigbur et al., 2011), the Simon task (Cohen, 2011; Cohen \& Ridderinkhof, 2013; Vissers, Ridderinkhof, Cohen, \& Slagter, 2018), and the Stroop task (Hanslmayr et al., 2008; Tang, Hu, \& Chen, 2013). Importantly, the conflict-related theta power was much increased in the alerting trials, compared to the no-tone trials, suggesting that the alerting-triggered increase of the flanker conflict entailed a greater involvement of the conflict processing mechanism.

In conclusion, the obtained results suggest that phasic alerting increases response conflict by modulations of sensorimotor processing at the stages prior to conflict detection and resolution, and that this increased conflict entails greater involvement of executive control. In other words, alerting affects both the emergence of conflict and conflict control. More specifically, alerting may affect conflict-related processing at three stages of the S-R pathway, first, by facilitating the automatic processing of incongruent stimuli, which increases interference within the S-R translation process; second, by enhancing the automatic activation of the incorrect repose via the direct S-R route, which increases the conflict between two mutually exclusive response programs; and third, by increasing the involvement of the mechanism of conflict detection and resolution.

\subsubsection{Conflict resolution and anterior N2}

One ERP component that has been associated with conflict resolution, but is not reported here, is the so-called conflict N2 - an enhancement of an anterior negative deflection often observed in the conflict trials (Kopp, Rist, \& Mattler, 1996; van Veen \& Carter, 2002). Typically, this $\mathrm{N} 2$ has its maximum at frontal midline (usually $\mathrm{FCz}$ ) at about $200-400 \mathrm{~ms}$ after target onset, and is measured in the go/nogo and the stop signal tasks as a marker of response inhibition (see Folstein and Van Petten, 2008, for a review). In the flanker task, the conflict N2 seems to be observed more often when e.g., the congruent-incongruent proportion is manipulated (Bartholow et al., 2005; Grützmann, Riesel, Klawohn, Kathmann, \& Endrass, 2014; Tillman \& Wiens, 2011) or no-go trials are included (Brydges et al., 2012; Heil, Osman, Wiegelmann, Rolke, \& Hennighausen, 2000; Kopp et al., 1996). In their recent study, Kałamała et al. (2017) have demonstrated that when the flanker task consists of only equiprobable congruent and incongruent trials (as in the present study), the conflict N2 either is absent or varies substantially in its topographical and temporal characteristics, compared to the typical anterior N2. They argued that in those cases, N2 may be explained as a frontal part of the P3 conflict effect (cf. Section 1.1), and in their own experiment $(N=52)$, they found neither the N2 conflict nor any underlying component that could correspond to the N2 conflict, despite having a high reliability of the ERPs and $100 \%$ power to found the true N2 effect. These findings suggest that resolution of the flanker conflict does not necessarily involve this specific process of response inhibition that is reflected in the anterior N2, and thus challenge the interpretation of $\mathrm{N} 2$ as an index of conflict processing (Kałamała et al., 2017; Tillman \& Wiens, 2011). Plausibly, the conflict-related modulation of midfrontal theta power (as measured in the present study) is a more valid electrophysiological index of flanker conflict processing, because it was found to be more closely and reliably linked to the behavioral conflict effect than the anterior N2 (Cohen \& Donner, 2013). Therefore, in the present study, we neither expected nor found the conflict N2 component. Instead, we have observed the conflict-related theta power modulation, and have replicated these results in our subsequent flanker task studies (in preparation).

\subsubsection{The response selection hypothesis}

The obtained results conform best to the hypothesis proposed by
Fischer et al. (2010). It should be mentioned here, however, that this hypothesis was initially introduced to explain the effect of accessory stimuli on response conflict specifically in the Simon task. Also, the effect of accessory stimuli on the incorrect response activation in the LRPs has already been observed, but again only in the Simon task (Böckler et al., 2011, although, as they noticed, the effect was not entirely consistent, thus needed a replication). The Simon and flanker effects are much alike, but they originate from different sources (Egner, 2008), which might make a difference regarding the processing stages at which alerting affects the conflict. Moreover, there is evidence that different conflict types involve at least partially different functional and neuronal mechanisms of conflict resolution (Dosenbach et al., 2006; Fan, Flombaum, McCandliss, Thomas, \& Posner, 2003; Nigbur et al., 2011), and that the effects of alerting on such control mechanisms may be task-specific (Soutschek, Müller, \& Schubert, 2013; Weinbach \& Henik, 2012). Therefore, the generalization of Fischer et al.'s (2010) and Böckler et al.'s (2011) findings and conclusions had remained in question. The present study shows that they can be generalized to the flanker task, and possibly to other contexts or situations of interaction between alerting and response conflict.

\subsubsection{The suppression hypothesis}

The present results, by showing that alerting entailed an increase, not decrease, of the brain activity associated with executive control, contradict both the hypothesis of alerting-triggered suppression of executive control (Callejas et al., 2005; Posner, 2008) and previous results suggesting that alerting affects only the emergence of conflict and not conflict control (Böckler et al., 2011, have found no effects of alerting on the anterior N2 component). Our findings are in line with some other recent studies. In their ERP experiment, Zani and Proverbio (2017) have found that the negative effect of alerting on flanker conflict was associated with an increase of a late midfrontal conflict-related negativity. This conflict negativity seems to originate in the MPFC (Zani \& Proverbio, 2017), same as the conflict-related theta oscillations. (Note, however, that we did not find any frontal incongruent-congruent differences in ERPs that would bear a resemblance to this conflict negativity nor any ERP equivalent of the conflict-related theta effect, which is in line with the notion that this theta modulation and the conflict-related frontal ERP effects reflect different neurocognitive processes, Cohen \& Donner, 2013.) Xuan et al. (2016), in turn, have reported fMRI results showing that alerting-conflict interaction in the flanker task was associated with an increased activation of the middle and inferior frontal gyri, and the anterior insular cortex - the nodes of the executive network (cf. Dosenbach, Fair, Cohen, Schlaggar, \& Petersen, 2008; Fan, 2014). Taken together, these results conform to our tentative conclusion that more resources of executive control are recruited to deal with the alerting-triggered increase of conflict, thereby arguing against the suppression hypothesis. Note, however, that these results do not contradict the basic notion that phasic alerting suppresses ongoing internal activity to facilitate perceptual processing of new stimuli (cf. Posner, 2008; Petersen \& Posner, 2012).

\subsubsection{The global processing and the early onset hypotheses}

In the present study, we found neither positive nor explicit negative evidence for the explanations of alerting-conflict interaction proposed by Weinbach and Henik (2012) and Nieuwenhuis and de Kleijn (2013). The latter may possibly still hold true, as it is not mutually exclusive with Fischer et al.'s account, it is based on a solid computational model, and recently it has gained some empirical support from a behavioral study by Schneider (2018a). One may also argue that the alerting effects on P3b latencies, as observed in the present study, correspond with the early onset hypotheses (notwithstanding, our results are generally better explained by the response selection hypothesis). The global processing hypothesis seems less plausible, since negative experimental evidence has recently been reported by Schneider (2018b). In this study, increasing stimulus spacing eliminated the effect of alerting on 
conflict, instead of enhancing it. Thus, the author proposed a spatial grouping hypothesis, predicting that alerting modifies processes of spatial grouping of stimuli within the spotlight of attention, rather than resizing the spotlight. Alerting is assumed here to strengthen spatial grouping, thereby increasing the difficulty of distinguishing between targets and distractors, which impairs response selection in the conflict conditions. This hypothesis would explain both Schneider's (2018b) and Weinbach and Henik's (2012) results. (Weinbach \& Henik, 2012, have shown that alerting increased conflict when the relevant and irrelevant information was spatially separated [flanker task], but not when the information was integrated into one object [Stroop task], and concluded that alerting expands the spotlight thereby increasing susceptibility for spatial distractors). Still, the spatial grouping hypothesis does not explain the results of the present study.

\subsection{Effects of SAT}

The SAT manipulation affected the conflict effect in ERRs, but not in RTs, which replicates the results of most previous SAT studies (Osman et al., 2000; Rinkenauer et al., 2004; Ullsperger et al., 2005; Wylie et al., 2009). In detail, the emphasis on accuracy decreased ERR in the incongruent condition only, most plausibly due to accuracy ceiling in the congruent condition, thus producing a smaller incongruent-congruent difference in the accuracy condition than in the speed condition. In RTs, SAT did not affect the conflict effect whatsoever: The speed gain resulted from speed strategy was the same in both the congruent and incongruent trials.

The behavioral effects of alerting were affected by SAT, but again only in the ERR measurement, and not in RTs. We found that accuracy strategy abolished both the main effect of alerting and the effect of alerting on conflict in the ERRs. This suggests that emphasis on accuracy may be an effective remedy for the harmful impact of phasic alerting on the accuracy of conflict resolution. In RTs, however, the alerting-conflict interaction was unaffected by SAT.

In the ERP analysis, we found that SAT modulated the effect of conflict in P3b amplitudes (described in the previous section): the incongruent-congruent difference was slightly larger in the speed condition than in the accuracy condition. This result corresponds with the higher accuracy in conflict trials under accuracy strategy, thus might be interpreted as evidence that activation of the correct S-R link in the conflict condition is easier when the system is tuned to a deeper, more careful and elaborate processing (see Bogacz et al., 2010; Heitz, 2014, for reviews of possible SAT mechanisms). In addition, the present results replicated two previously observed effects of SAT: Speed emphasis was associated with a general increase of the P3b amplitudes (cf. Pfefferbaum et al., 1983) and with a general increase of the amplitudes of the correct activations in LRPs (cf. Sangals et al., 2002), as compared to accuracy emphasis.

Importantly, both the ERP and time-frequency analyses showed no effects of SAT on the alerting-conflict interaction, in line with the present RT results. Taken together, the RT and EEG results suggest that the effects of alerting on response conflict cannot be interpreted, let alone explained, in terms of speed-accuracy tradeoff.

\subsection{Concluding remarks}

The results of the present study suggest that exogenous phasic alerting affects processing of response conflict at three stages of the S-R pathway: First, by facilitating the automatic processing of incongruent stimuli, which increases interference within the S-R translation process. Second, by enhancing the automatic activation of the incorrect response programs. Third, by increasing the involvement of executive control in response to the increased conflict. In conclusion, alerting affects both the emergence of conflict and conflict control, and these effects could not be explained as an alerting-triggered imbalance of speed-accuracy tradeoff.

\section{Acknowledgment}

This work was supported by Grant 2016/22/E/HS6/00139 awarded from the National Science Centre of Poland to D.A.

\section{References}

Asanowicz, D., \& Marzecová, A. (2017). Differential effects of phasic and tonic alerting on the efficiency of executive attention. Acta Psychologica, 176, 58-70.

Bartholow, B. D., Pearson, M. A., Dickter, C. L., Sher, K. J., Fabiani, M., \& Gratton, G. (2005). Strategic control and medial frontal negativity: Beyond errors and response conflict. Psychophysiology, 42(1), 33-42.

Böckler, A., Alpay, G., \& Stürmer, B. (2011). Accessory stimuli affect the emergence of conflict, not conflict control: A Simon-task ERP study. Experimental Psychology, 58(2), 102-109.

Bogacz, R., Wagenmakers, E. J., Forstmann, B. U., \& Nieuwenhuis, S. (2010). The neural basis of the speed-accuracy tradeoff. Trends in Cognitive Science, 33(1), 10-16.

Botvinick, M. M., Braver, T. S., Barch, D. M., Carter, C. S., \& Cohen, J. D. (2001). Conflict monitoring and cognitive control. Psychological Review, 108(3), 624-652.

Brydges, C. R., Clunies-Ross, K., Clohessy, M., Lo, Z. L., Nguyen, A., Rousset, C., ... Fox, A M. (2012). Dissociable components of cognitive control: An event-related potential (ERP) study of response inhibition and interference suppression. PloS One, 7(3), 344-382.

Callejas, A., Lupiáñez, J., \& Tudela, P. (2004). The three attentional networks: On their independence and interactions. Brain and Cognition, 54(3), 225-227.

Callejas, A., Lupiáñez, J., Funes, M., \& Tudela, P. (2005). Modulations among the alerting, orienting and executive control networks. Experimental Brain Research, 167(1), 27-37.

Cavanagh, J. F., \& Frank, M. J. (2014). Frontal theta as a mechanism for cognitive control. Trends in Cognitive Sciences, 18, 414-421.

Cavanagh, J. F., Cohen, M. X., \& Allen, J. J. B. (2009). Prelude to and resolution of an error: EEG phase synchrony reveals cognitive control dynamics during action monitoring. Journal of Neuroscience, 29(1), 98-105.

Cohen, M. X. (2011). Error-related medial frontal theta activity predicts cingulate-related structural connectivity. NeuroImage, 55(3), 1373-1383.

Cohen, M. X. (2017). MATLAB for brain and cognitive scientists. Cambridge, MA: MIT Press.

Cohen, M. X. (2014a). A neural microcircuit for cognitive conflict detection and signaling. Trends in Neurosciences, 37, 480-490.

Cohen, M. X. (2014b). Analyzing neural time series data: Theory and practice. Cambridge, MA: MIT Press.

Cohen, M. X., \& Cavanagh, J. F. (2011). Single-trial regression elucidates the role of prefrontal theta oscillations in response conflict. Frontiers in Psychology, 2(30), 1-12.

Cohen, M. X., \& Donner, T. H. (2013). Midfrontal conflict-related theta-band power reflects neural oscillations that predict behavior. Journal of Neurophysiology, 110(12), 2752-2763.

Cohen, M. X., \& Ridderinkhof, K. R. (2013). EEG source reconstruction reveals frontalparietal dynamics of spatial conflict processing. PloS One, 8(2), e57293.

Coles, M. G. (1989). Modern mind-brain reading: Psychophysiology, physiology, and cognition. Psychophysiology, 26(3), 251-269.

De Jong, R., Liang, C. C., \& Lauber, E. (1994). Conditional and unconditional automaticity: A dual-process model of effects of spatial stimulus-response correspondence. Journal of Experimental Psychology. Human Perception and Performance, 20(4), 731-750.

Dosenbach, N. U., Fair, D. A., Cohen, A. L., Schlaggar, B. L., \& Petersen, S. E. (2008). A dual-networks architecture of top-down control. Trends in Cognitive Sciences, 12(3), 99-105.

Dosenbach, N. U., Visscher, K. M., Palmer, E. D., Miezin, F. M., Wenger, K. K., Kang, H. C., .. Petersen, S. E. (2006). A core system for the implementation of task sets. Neuron, 50(5), 799-812.

Egner, T. (2008). Multiple conflict-driven control mechanisms in the human brain. Trends in Cognitive Sciences, 12(10), 374-380.

Eimer, M. (1998). The lateralized readiness potential as an on-line measure of central response activation processes. Behavior Research Methods, Instruments, \& Computers, 30(1), 146-156.

Eimer, M., Hommel, B., \& Prinz, W. (1995). S-R compatibility and response selection. Acta Psychologica, 90(1), 301-313.

Eriksen, B. A., \& Eriksen, C. W. (1974). Effects of noise letters upon the identification of a target letter in a nonsearch task. Perception \& Psychophysics, 16(1), 143-149.

Fan, J. (2014). An information theory account of cognitive control. Frontiers in Human Neuroscience, 8(680), 1-16.

Fan, J., Flombaum, J., McCandliss, B., Thomas, K., \& Posner, M. (2003). Cognitive and brain consequences of conflict. NeuroImage, 18(1), 42-57.

Fischer, R., Plessow, F., \& Kiesel, A. (2010). Auditory warning signals affect mechanisms of response selection: Evidence from a Simon task. Experimental Psychology, 57(2), 89-97.

Fischer, R., Plessow, F., \& Kiesel, A. (2012). The effects of alerting signals in action control: Activation of S-R associations or inhibition of executive control processes? Psychological Research, 76(3), 317-328.

Fischer, R., Plessow, F., \& Ruge, H. (2013). Priming of visual cortex by temporal attention? The effects of temporal predictability on stimulus (-specific) processing in early visual cortical areas. NeuroImage, 66, 261-269.

Folstein, J. R., \& Van Petten, C. (2008). Influence of cognitive control and mismatch on the N2 component of the ERP: A review. Psychophysiology, 45(1), 152-170.

Fossella, J., Sommer, T., Fan, J., Wu, Y., Swanson, J., Pfaff, D., ... Posner, M. (2002). 
Assessing the molecular genetics of attention networks. BMC Neuroscience, 3, 14.

Gratton, G., Coles, M. G., \& Donchin, E. (1983). A new method for off-line removal of ocular artifact. Electroencephalography and Clinical Neurophysiology, 55(4), 468-484.

Gratton, G., Coles, M. G., \& Donchin, E. (1992). Optimizing the use of information: Strategic control of activation of responses. Journal of Experimental Psychology General, 121(4), 480-506.

Gratton, G., Coles, M. G., Sirevaag, E. J., Eriksen, C. W., \& Donchin, E. (1988). Pre- and poststimulus activation of response channels: A psychophysiological analysis. Journal of Experimental Psychology. Human Perception and Performance, 14(3), 331-344.

Grützmann, R., Riesel, A., Klawohn, J., Kathmann, N., \& Endrass, T. (2014). Complementary modulation of N2 and CRN by conflict frequency. Psychophysiology, 51(8), 761-772.

Hackley, S. A., \& Valle-Inclán, F. (1998). Automatic alerting does not speed late motoric processes in a reaction-time task. Nature, 391(6669), 786-788.

Hackley, S. A., \& Valle-Inclán, F. (1999). Accessory stimulus effects on response selection: Does arousal speed decision making? Journal of Cognitive Neuroscience, 11(3), 321-329.

Hackley, S. A., \& Valle-Inclán, F. (2003). Which stages of processing are speeded by a warning signal? Biological Psychology, 64(1-2), 27-45.

Hackley, S. A., Langner, R., Rolke, B., Erb, M., Grodd, W., \& Ulrich, R. (2009). Separation of phasic arousal and expectancy effects in a speeded reaction time task via fMRI. Psychophysiology, 46(1), 163-171.

Hanslmayr, S., Pastötter, B., Bäuml, K. H., Gruber, S., Wimber, M., \& Klimesch, W. (2008). The electrophysiological dynamics of interference during the Stroop task. Journal of Cognitive Neuroscience, 20(2), 215-225.

Heil, M., Osman, A., Wiegelmann, J., Rolke, B., \& Hennighausen, E. (2000). N200 in the Eriksen-task: Inhibitory executive process? Journal of Psychophysiology, 14(4), 218-225.

Heitz, R. P. (2014). The speed-accuracy tradeoff: History, physiology, methodology, and behavior. Frontiers in Neuroscience, 8(150), 1-19.

Hommel, B. (2004). Event files: Feature binding in and across perception and action. Trends in Cognitive Sciences, 8(11), 494-500.

Hopf, J. M., Vogel, E., Woodman, G., Heinze, H. J., \& Luck, S. J. (2002). Localizing visual discrimination processes in time and space. Journal of Neurophysiology, 88(4), 2088-2095.

Ishigami, Y., \& Klein, R. M. (2010). Repeated measurement of the components of attention using two versions of the attention network test (ANT): Stability, isolability, robustness, and reliability. Journal of Neuroscience Methods, 190(1), 117-128.

Jepma, M., Wagenmakers, E. J., Band, G. P., \& Nieuwenhuis, S. (2009). The effects of accessory stimuli on information processing: Evidence from electrophysiology and a diffusion model analysis. Journal of Cognitive Neuroscience, 21(5), 847-864.

Kałamała, P., Drożdżowicz, A., Szewczyk, J., Marzecová, A., \& Wodniecka, Z. (2018). Task strategy may contribute to performance differences between monolinguals and bilinguals in cognitive control tasks: ERP evidence. Journal of Neurolinguistics, 46, 78-92.

Kałamała, P., Szewczyk, J., Senderecka, M., \& Wodniecka, Z. (2017). Flanker task with equiprobable congruent and incongruent conditions does not elicit the conflict N2. Psychophysiology, 55(2), e12980.

Kiesel, A., Miller, J., Jolicoeur, P., \& Brisson, B. (2008). Measurement of ERP latency differences: A comparison of single-participant and jackknife-based scoring methods. Psychophysiology, 45(2), 250-274.

Kopp, B., Rist, F., \& Mattler, U. (1996). N200 in the flanker task as a neurobehavioral tool for investigating executive control. Psychophysiology, 33(3), 282-294.

Kusnir, F., Chica, A. B., Mitsumasu, M. A., \& Bartolomeo, P. (2011). Phasic auditory alerting improves visual conscious perception. Consciousness and Cognition, 20(4), 1201-1210.

Maris, E., \& Oostenveld, R. (2007). Nonparametric statistical testing of EEG- and MEGdata. Journal of Neuroscience Methods, 164(1), 177-190.

Miller, J. (1991). The flanker compatibility effect as a function of visual angle, attentional focus, visual transients, and perceptual load: A search for boundary conditions. Perception \& Psychophysics, 49(3), 270-288.

Miller, J., Patterson, T., \& Ulrich, R. (1998). Jackknife-based method for measuring LRP onset latency differences. Psychophysiology, 35(1), 99-115.

Neuhaus, A. H., Urbanek, C., Opgen-Rhein, C., Hahn, E., Ta, T. M., Koehler, S., ... Dettling, M. (2010). Event-related potentials associated with attention network test. International Journal of Psychophysiology, 76(2), 72-79.

Nieuwenhuis, S., \& de Kleijn, R. (2013). The impact of alertness on cognitive control. Journal of Experimental Psychology. Human Perception and Performance, 39(6), 1797-1801.

Nigbur, R., Ivanova, G., \& Stürmer, B. (2011). Theta power as a marker for cognitive interference. Clinical Neurophysiology, 122(11), 2185-2194.

Osman, A., Lou, L., Muller-Gethmann, H., Rinkenauer, G., Mattes, S., \& Ulrich, R. (2000). Mechanisms of speed-accuracy tradeoff: Evidence from covert motor processes. Biological Psychology, 51(2-3), 173-199.

Pastötter, B., Hanslmayr, S., \& Bauml, K. H. T. (2010). Conflict processing in the anterior cingulate cortex constrains response priming. NeuroImage, 50(4), 1599-1605.

Petersen, S. E., \& Posner, M. I. (2012). The attention system of the human brain: 20 years after. Annual Review of Neuroscience, 35, 73-89.

Petersen, A., Petersen, A. H., Bundesen, C., Vangkilde, S., \& Habekost, T. (2017). The effect of phasic auditory alerting on visual perception. Cognition, 165, 73-81.

Pfefferbaum, A., Ford, J., Johnson, R., Wenegrat, B., \& Kopell, B. S. (1983). Manipulation of P3 latency: Speed vs. accuracy instructions. Electroencephalography and Clinical Neurophysiology, 55(2), 188-197.

Posner, M. I. (1994). Attention: The mechanisms of consciousness. Proceedings of the National Academy of Sciences of the United States of America, 91(16), 7398-7403.

Posner, M. I. (2008). Measuring alertness. Annals of the New York Academy of Sciences,
1129, 193-199.

Posner, M. I., \& Rothbart, M. K. (2007). Research on attention networks as a model for the integration of psychological science. Annual Review of Psychology, 58, 1-23.

Posner, M. I., Klein, R., Summers, J., \& Buggie, S. (1973). On the selection of signals. Memory \& Cognition, 1(1), 2-12.

Posner, M. I., Nissen, M. J., \& Klein, R. M. (1976). Visual dominance: An informationprocessing account of its origins and significance. Psychological Review, 83(2), $157-171$.

Pratt, H. (2011). Sensory ERP components. In E. S. Kappenman, \& S. J. Luck (Eds.). The Oxford handbook of event-related potential components. Oxford University Press.

Pritchard, W. S., Houlihan, M. E., \& Robinson, J. H. (1999). P300 and response selection: A new look using independent-components analysis. Brain Topography, 12(1), 31-37.

Ridderinkhof, K. R., Ullsperger, M., Crone, E. A., \& Nieuwenhuis, S. (2004). The role of the medial frontal cortex in cognitive control. Science, 306, 443-447.

Ridderinkhof, R. K., van den Wildenberg, W. P. M., \& Wylie, S. A. (2012). Action control in times of conflict. Analysis of reaction time distributions in healthy and clinical populations. In M. I. Posner (Ed.). Cognitive neuroscience of attention (pp. 409-420). (second edition). New York, London: Guilford Press.

Rinkenauer, G., Osman, A., Ulrich, R., Muller-Gethmann, H., \& Mattes, S. (2004). On the locus of speed-accuracy trade-off in reaction time: Inferences from the lateralized readiness potential. Journal of Experimental Psychology: General, 133(2), 261-282.

Sangals, J., Sommer, W., \& Leuthold, H. (2002). Influences of presentation mode and time pressure on the utilisation of advance information in response preparation. Acta Psychologica, 109(1), 1-24.

Schneider, D. W. (2018a). Alertness and cognitive control: Testing the early onset hypothesis. Journal of Experimental Psychology: Human Perception and Performance, 44(5), 756-766.

Schneider, D. W. (2018b). Alertness and cognitive control: Toward a spatial grouping hypothesis. Attention, Perception, and Psychophysics, 80(4), 913-928.

Simon, J. R. (1969). Reactions toward the source of stimulation. Journal of Experimental Psychology, 81(1), 174-176.

Smid, H. G., Mulder, G., \& Mulder, L. J. (1990). Selective response activation can begin before stimulus recognition is complete: A psychophysiological and error analysis of continuous flow. Acta Psychologica, 74(2), 169-210.

Smulders, F. T. Y., \& Miller, J. O. (2012). The lateralized readiness potential. Oxford library of psychology: The Oxford handbook of event-related potential components. New York, NY, US: Oxford University Press209-229.

Soutschek, A., Müller, H. J., \& Schubert, T. (2013). Conflict-specific effects of accessory stimuli on cognitive control in the Stroop task and the Simon task. Experimental Psychology, 60(2), 140-147.

Sternberg, S. (1969). The discovery of processing stages: Extensions of Donders' method. Acta Psychologica, 30, 276-315.

Stroop, J. R. (1935). Studies of interference in serial verbal reactions. Journal of Experimental Psychology, 18, 643-662.

Tang, D., Hu, L., \& Chen, A. (2013). The neural oscillations of conflict adaptation in the human frontal region. Biological Psychology, 93(3), 364-372.

Tillman, C. M., \& Wiens, S. (2011). Behavioral and ERP indices of response conflict in Stroop and flanker tasks. Psychophysiology, 48(10), 1405-1411.

Twomey, D. M., Murphy, P. R., Kelly, S. P., \& O'Connell, R. G. (2015). The classic P300 encodes a build-to-threshold decision variable. The European Journal of Neuroscience, 42(1), 1636-1643.

Ullsperger, M., Bylsma, L. M., \& Botvinick, M. M. (2005). The conflict adaptation effect: It's not just priming. Cognitive, Affective \& Behavioral Neuroscience, 5(4), 467-472.

Ulrich, R., \& Miller, J. (2001). Using the jackknife-based scoring method for measuring LRP onset effects in factorial designs. Psychophysiology, 38(5), 816-827.

Valle-Inclán, F. (1996). The Simon effect and its reversal studied with event-related potentials. International Journal of Psychophysiology, 23(1-2), 41-53.

van der Lubbe, R. H., Jaśkowski, P., Wauschkuhn, B., \& Verleger, R. (2001). Influence of time pressure in a simple response task, a choice-by-location task, and the Simon task. Journal of Psychophysiology, 15(4), 241-255.

van Veen, V., \& Carter, C. S. (2002). The timing of action-monitoring processes in the anterior cingulate cortex. Journal of Cognitive Neuroscience, 14(4), 593-602.

Verleger, R. (1997). On the utility of P3 latency as an index of mental chronometry. Psychophysiology, 34(2), 131-156.

Verleger, R., Hagenah, J., Weiss, M., Ewers, T., Heberlein, I., Pramstaller, P. P., ... Klein, C. (2010). Responsiveness to distracting stimuli, though increased in Parkinson's disease, is decreased in asymptomatic PINK1 and Parkin mutation carriers. Neuropsychologia, 48(2), 467-476.

Verleger, R., Jaśkowski, P., \& Wascher, E. (2005). Evidence for an integrative role of P3b in linking reaction to perception. Journal of Psychophysiology, 19(3), 165.

Verleger, R., Kuniecki, M., Möller, F., Fritzmannova, M., \& Siebner, H. R. (2009). On how the motor cortices resolve an inter-hemispheric response conflict: An event-related EEG potential-guided TMS study of the flankers task. The European Journal of Neuroscience, 30(2), 318-326.

Verleger, R., Metzner, M. F., Ouyang, G., Śmigasiewicz, K., \& Zhou, C. (2014). Testing the stimulus-to-response bridging function of the oddball-P3 by delayed response signals and residue iteration decomposition (RIDE). NeuroImage, 100C, 271-280.

Verleger, R., Śmigasiewicz, K., \& Möller, F. (2011). Mechanisms underlying the left visual-field advantage in the dual stream RSVP task: Evidence from N2pc, P3, and distractor-evoked VEPs. Psychophysiology, 48(8), 1096-1106.

Verleger, R., Asanowicz, D., Werner, L., \& Śmigasiewicz, K. (2015). Biased odds for heads or tails: Outcome-evoked P3 depends on frequencies of guesses. Psychophysiology, 52(8), 1048-1058.

Verleger, R., Hamann, L. M., Asanowicz, D., \& Śmigasiewicz, K. (2015). Testing the S-R link hypothesis of P3b: The oddball effect on S1-evoked P3 gets reduced by increased task relevance of S2. Biological Psychology, 108, 25-35. 
Vissers, M. E., Ridderinkhof, K. R., Cohen, M. X., \& Slagter, H. A. (2018). Oscillatory mechanisms of response conflict elicited by color and motion direction: An individual differences approach. Journal of Cognitive Neuroscience, 30(4), 468-481.

Vogel, E. K., \& Luck, S. J. (2000). The visual N1 component as an index of a discrimination process. Psychophysiology, 37(2), 190-203.

Weinbach, N., \& Henik, A. (2012). The relationship between alertness and executive control. Journal of Experimental Psychology. Human Perception and Performance, 38(6), 1530-1540.

Weinbach, N., \& Henik, A. (2013). The interaction between alerting and executive control: Dissociating phasic arousal and temporal expectancy. Attention, Perception \& Psychophysics, 75(7), 1374-1381.

Wiegand, I., Petersen, A., Finke, K., Bundesen, C., Lansner, J., \& Habekost, T. (2017). Behavioral and brain measures of phasic alerting effects on visual attention. Frontiers in Human Neuroscience, 11(176), 1-11.
Wylie, S. A., van den Wildenberg, W. P., Ridderinkhof, K. R., Bashore, T. R., Powell, V. D., Manning, C. A., ... Wooten, G. F. (2009). The effect of speed-accuracy strategy on response interference control in Parkinson's disease. Neuropsychologia, 47(8-9), 1844-1853.

Xuan, B., Mackie, M. A., Spagna, A., Wu, T., Tian, Y., Hof, P. R., ... Fan, J. (2016). The activation of interactive attentional networks. Neuroimage, 129, 308-319.

Yanaka, H. T., Saito, D. N., Uchiyama, Y., \& Sadato, N. (2010). Neural substrates of phasic alertness: A functional magnetic resonance imaging study. Neuroscience Research, 68(1), 51-58.

Yoshida, Y., Tanabe, H. C., Hayashi, M. J., Kawamichi, H., Kochiyama, T., \& Sadato, N. (2013). The neural substrates of the warning effect: A functional magnetic resonance imaging study. Neuroscience Research, 76(4), 230-239.

Zani, A., \& Proverbio, A. M. (2017). How voluntary orienting of attention and alerting modulate costs of conflict processing. Scientific Reports, 7(46701), 1-14. 